\author{
可控释放一氧化碳的纳米材料及其生物医学应用 \\ 张晓蕾 $a, b$ \\ 田甘c \\ 张潇*, \\ 王清*, $a$ \\ 谷战军 $b$ \\ $\left({ }^{a}\right.$ 山东科技大学 土木工程与建筑学院 青岛 266590) \\ $\left({ }^{b}\right.$ 中国科学院高能物理研究所 中国科学院纳米生物效应与安全性重点实验室 北京 100049) \\ ( c 第三军医大学第一附属医院病理学研究所\&西南癌症中心 肿瘤免疫病理学教育部重点实验室 重庆 400038)
}

\begin{abstract}
摘要 一氧化碳 $(\mathrm{CO})$ 是一种内源性气体信使分子, 具有广泛而复杂的生理学功能. $\mathrm{CO}$ 分子的生理学效应与其浓度、位 置和作用时间密切相关. 而现有的一氧化碳供体普遍存在着稳定性较差, 剂量难以把控, 缺乏靶向性以及对正常细胞 和组织器官具有潜在的毒副作用等问题, 限制了其进一步的应用. 随着纳米科学技术的迅速发展, 国内外研究者们构 建出一系列能够实现可控释放 CO 的多功能纳米材料, 并将其用于生物医学领域. 结合纳米材料自身独特的性能优势, 分类介绍了多种内源性/外源性刺激响应型 $\mathrm{CO}$ 控释纳米材料, 并概述了可控释放 $\mathrm{CO}$ 的纳米药物在抑制炎症反应、抗 菌和肿瘤治疗等生物医学领域的应用, 最后对 $\mathrm{CO}$ 控释纳米材料在生物医学领域面临的挑战和发展前景进行了总结和 讨论.
\end{abstract}

关键词 一氧化碳; 纳米材料; 可控释放; 内源性及外源性刺激控释; 一氧化碳纳米药物

\title{
Controlled Release of Carbon Monoxide Based on Nanomaterials and Their Biomedical Applications
}

\author{
Zhang, Xiaolei ${ }^{a, b} \quad$ Tian, Gan $^{c} \quad$ Zhang, Xiao*,b Wang, Qing* ${ }^{*, a}$ Gu, Zhanjun ${ }^{b}$ \\ $\left({ }^{a}\right.$ College of Civil Engineering and Architecture, Shandong University of Science and Technology, Qingdao 266590) \\ $\left({ }^{b}\right.$ CAS Key Laboratory for Biomedical Effects of Nanomaterials and Nanosafety, Institute of High Energy Physics, Chinese \\ Academy of Sciences, Beijing 100049) \\ ( ${ }^{c}$ Institute of Pathology and Southwest Cancer Center, First Affiliated Hospital, Third Military Medical University, and Key \\ Laboratory of Tumor Immunopathology, Ministry of Education of China, Chongqing 400038)
}

\begin{abstract}
In recent years, the use of gas therapy has been more and more concerned by researchers in biomedical applications. Carbon monoxide ( $\mathrm{CO})$ is a diatomic gas messenger molecule with the function of transmitting intercellular information and regulating cellular signals. CO is found to play an extremely important physiological role in multiple systems, including cardiovascular system, nervous system, immune system, endocrine system and respiratory system, cancer therapy, coagulation and fibrinolysis system, organ transplantation and preservation, and so on. The biological functions of carbon monoxide molecule greatly depend on the its concentration, position, and duration. However, the existing carbon monoxide donors including $\mathrm{Mn}_{2}(\mathrm{CO})_{10}, \mathrm{Ru}_{2} \mathrm{Cl}_{4}(\mathrm{CO})_{6}, \mathrm{Ru}(\mathrm{CO})_{3} \mathrm{Cl}$ (glycinato), CORM-F, CORM-A1 have some disadvantages, such as poor stability, difficulties in dose control, lack of targeting, potential toxic and side effects on normal cells and tissues, which limited their further applications. How to control the concentration of carbon monoxide in the specific region has always been a big challenge in the field of biomedical applications. With the rapid development of nanoscience and technology, researchers have constructed a series of multifunctional carbon monoxide releasing nanomaterials, provided a new idea for $\mathrm{CO}$ controlled release, and applied them in the field of biomedicine. In this paper, several kinds of endogenous/exogenous stimulus-responsive $\mathrm{CO}$ releasing nanomaterials with the unique advantages are introduced based on the stimuli source. Then, the applications of these controlled CO releasing nanomaterials in biomedical fields, such as inhibiting inflammation, anti-bacterial and cancer therapy, are summarized. Finally, the challenges and prospects of $\mathrm{CO}$ releasing nanomaterials are discussed. Keywords carbon monoxide; nanomaterials; controlled release; endogenous/exogenous stimulation; carbon monoxide nanodrugs
\end{abstract}

\footnotetext{
*E-mail: zhangx89@ihep.ac.cn; qwang@sdust.edu.cn

Received December 17, 2018; published February 8, 2019

Project supported by the National Basic Research Programs of China (Nos. 2016YFA0201600, 2016YFA0202104), the National Natural Science Foundation of China (Nos. 51822207, 51772292, 31571015, 11621505, 11435002, 81703071) and Youth Innovation Promotion Association of Chinese Academy of Sciences (No. 2013007) and Chongqing Basic and Frontier Research Program (No. cstc2016jcyjA0279) and Military Medical Science and Technology Innovation Program of Southwest Hospital (Nos. SWH2016LHJC-07, SWH2016JCYB-01 and SWH2017YQPY-03).

项目受国家重点基础研究发展计划(Nos. 2016YFA0201600, 2016YFA0202104), 国家自然科学基金(Nos. 51822207, 51772292, 31571015, 11621505, $11435002,81703071) 及 中$ 国科学院青年创新促进会基金(No. 2013007), 重庆市基础与前沿研究项目(No. cstc2016jcyjA0279)、西南医院军事医学科技 创新项目(Nos. SWH2016LHJC-07、SWH2016JCYB-01、SWH2017YQPY-03)资助.
} 


\section{1 引言}

自 20 世纪初期起, 一氧化碳(Carbon monoxide, CO) 被公认为是一种无色无味、污染环境、损害健康的有毒 气体 ${ }^{[1 \sim 3]} .1968$ 年, Schmid 等发现生物体内能够表达血 红素加氧酶, 而这种酶在生物体内可以持续地催化产生 $\mathrm{CO}^{[4 \sim 7]}$. 由此, 研究者们意识到 $\mathrm{CO}$ 可能有着非常重要 的生理作用和功能. 随着研究进一步的深入, 研究人员 发现 $\mathrm{CO}$ 可以作为一种信使分子, 参与调控神经系统、 心血管系统和免疫系统中的一些生理过程 ${ }^{[8-12]}$. 如今, 越来越多的研究揭示了 $\mathrm{CO}$ 的生理学功能, 并将其应用 于多种疾病的治疗中, 包括炎症、消化道疾病、动脉粥 状硬化、中风、甚至癌症等 ${ }^{[11,13,14]}$.

内源性的 CO 主要来源于血红素加氧酶(Haem oxygenase, HO)催化分解血红素产生 ${ }^{[15 ~ 17]}$. 而对于外源性 的 $\mathrm{CO}$ 来说, 最简单的给予方式是直接吸入 $\mathrm{CO}$ 气体. 但 是 $\mathrm{CO}$ 分子与血红蛋白有较强的亲和力 (是氧气与血红 蛋白结合能力的 200 倍以上), 直接吸入 CO 容易使得血 红蛋白失去输运氧气的能力, 进而引起严重的后果 ${ }^{[3,18]}$. 因此, 研究人员利用外源性 CO 供体或输运体系, 在特 定的位置和时间释放定量的 CO 分子, 以此达到良好的 治疗效果以及避免副作用的产生 ${ }^{[19]}$. 目前, $\mathrm{CO}$ 供体分 子(carbon monoxide releasing molecules, CORMs)的研究 相对成熟, 部分供体已经商品化 ${ }^{[11,14,20 ~ 22]}$, 例如 $\mathrm{Mn}_{2}(\mathrm{CO})_{10}, \quad \mathrm{Ru}_{2} \mathrm{Cl}_{4}(\mathrm{CO})_{6}, \quad \mathrm{Ru}(\mathrm{CO})_{3} \mathrm{Cl}$ (glycinato) 等. CORMs 通常包括一个过渡金属中心, 如 $\mathrm{Fe} 、 \mathrm{Mn} 、 \mathrm{Ni}$ 、 $\mathrm{Co} 、 \mathrm{Ru}$ 等重金属, 这些重金属被不同数目的羰基包围, 形成不同类型的金属羰基络合物 ${ }^{[1]}$. CORMs 种类较多, 主要包括 CORM-1 $\left(\mathrm{Mn}_{2}(\mathrm{CO})_{10}\right) 、 \mathrm{CORM}-2\left(\mathrm{Ru}_{2} \mathrm{Cl}_{4}(\mathrm{CO})_{6}\right)$ 、 CORM-3( $\mathrm{Ru}(\mathrm{CO})_{3} \mathrm{Cl}$ (glycinato))、CORM- $\mathrm{F}\left(\left[\mathrm{Fe}(\mathrm{CO})_{5}\right]\right.$, 五 羰基铁衍生物和 CORM- $\mathrm{A} 1\left(\mathrm{Na}_{2}\left[\mathrm{H}_{3} \mathrm{BCO}_{2}\right]\right)$ 及其衍生物 $\left(\mathrm{Na}\left[\mathrm{H}_{3} \mathrm{BCO}(\right.\right.$ morph $\left.\left.)\right]\right) 、 \mathrm{Na}\left[\mathrm{H}_{3} \mathrm{BCO}(\mathrm{gly})\right] 、 \mathrm{Na}\left[\mathrm{H}_{3} \mathrm{BCO}-\right.$ (hist)]等. 其中, CORM-1 是最早被使用的 $\mathrm{CO}$ 供体, 该 分子不溶于水可溶于二甲基亚砜(DMSO), 且要在冷光 源下照射才能够释放 CO, 应用局限性较大. CORM-2 分 子同样不溶于水, 可溶于 DMSO, 但其不需要冷光源就 能自发释放 CO. CORM-3 是一种水溶且生物安全性较 好的 CO 供体分子. 其在酸性条件下能够保持稳定, 而 在中性条件或碱性条件下释放 CO. CORM-F, CORM-A1 及其衍生物都是水溶性的 $\mathrm{CO}$ 供体, 能够缓慢释放 CO. 但是, 这些已有的 CORMs 在实际使用时仍然存在许多 局限, 如稳定性差、缺乏靶向性、药物作用时间短、剂 量难以掌控等. 仅仅通过有机合成或官能团修饰的方 法, 难以得到具有理想性能的 $\mathrm{CO}$ 供体. 而近年来纳米 科学的迅猛发展, 为一氧化碳的可控释放提供了新的思 路. 越来越多的研究者开始尝试将 $\mathrm{CO}$ 供体与功能性纳 米材料结合 ${ }^{[23]}$, 构建具有刺激响应能力的 CO 输运纳米 载体, 以此实现 $\mathrm{CO}$ 的可控释放 ${ }^{[24]}$. 这些功能性纳米材 料主要包括介孔二氧化硅纳米球、二维纳米材料、上转
换纳米材料、有机金属框架纳米材料、中空笼形蛋白、 高分子聚合物等. 其中, 介孔二氧化硅、二维纳米材料 和有机金属框架纳米材料具有比表面积大、稳定性好和 较高的药物负载率等特性, 一直备受青崃. 中空笼形蛋 白和高分子聚合物纳米材料因其水溶性好, 结构可塑性 和生物相容性好的特点而被广泛用于纳米颗粒的修饰、 药物输运和靶向释放等领域. 而上转换纳米材料等一些 具有光功能特性的材料因其能够对外界刺激响应而备 受欢迎. 本综述主要介绍了近期在基于纳米材料的 $\mathrm{CO}$ 可控输运体系及其在生物应用领域的研究进展. 首先, 我们分类介绍多种刺激响应型 $\mathrm{CO}$ 控释纳米材料. 然后, 系统展示 CO 控释纳米材料在生物医学领域的应用. 最 后, 我们总结了 $\mathrm{CO}$ 控释纳米材料在生物医学领域的前 景和面临的挑战.

\section{2 刺激响应型一氧化碳控释纳米材料}

由于 $\mathrm{CO}$ 的生物学效应存在浓度依赖性 ${ }^{[1,20]}$, 如何 在生物体内精准地定量释放一氧化碳分子成为 $\mathrm{CO}$ 临床 应用的关键问题. 近年来, 研究人员致力于设计合成刺 激响应型 CO 控释材料, 在空间上和时间上实现精准的 $\mathrm{CO}$ 释放, 以此减少潜在的毒副作用 ${ }^{[25 ~ 27]}$. 根据刺激源 的不同, 刺激响应型 $\mathrm{CO}$ 控释纳米材料可以分为内源性 响应 $\left(\mathrm{pH} 、 \mathrm{H}_{2} \mathrm{O}_{2} \text { 等 }\right)^{[23,28,29]}$ 和外源性响应(光、磁场等 $)^{[30]}$ 两类.

\section{$2.1 \mathrm{pH}$}

在生物体内, 各个器官、组织甚至不同细胞器之间 的 $\mathrm{pH}$ 值都有所差别 ${ }^{[31 ~ 34]}$. 相对于正常组织, 发炎、感 染、恶性肿瘤等病变部位的 $\mathrm{pH}$ 值会发生明显的变化, 这 使得 $\mathrm{pH}$ 响应型控释纳米材料在生物医药领域具有诱人 的应用前景 ${ }^{[1,28,33,35 \sim 44]}$. Motterlini 等 ${ }^{[45]}$ 发现水溶性的 CORM-A1( $\left.\mathrm{Na}_{2}\left[\mathrm{H}_{3} \mathrm{BCO}_{2}\right]\right)$ 在碱性条件下近乎稳定, 而在 酸性条件下可以快速释放 $\mathrm{CO}$, 可以作为一种 $\mathrm{pH}$ 响应型 $\mathrm{CO}$ 供体应用于生物医学领域. 借助纳米材料比表面积 大、官能团丰富、易于修饰的特点, 研究者们设想将 $\mathrm{pH}$ 响应型 $\mathrm{CO}$ 供体负载于纳米颗粒表面或利用纳米颗粒构 建 $\mathrm{pH}$ 响应型纳米结构 ${ }^{[46,47]}$, 以此实现 $\mathrm{CO}$ 的可控释放.

Boyer 等 ${ }^{[88]}$ 设计合成了一种双亲性的嵌段共聚物 (P(OEGA)-b-P(4VP)), 然后将 CO 供体 CORM-2 修饰在 该嵌段共聚物上, 通过自组装的方式形成纳米胶束 (P(OEGA)-b-P(4VP-CORM-2)). 与单独的 CORM-2 相 比, 这种负载 CORM-2 分子的纳米胶束的水溶性得到显 著的提升. 由于负载的 CORM-2 分子处于纳米颗粒内部 疏水结构区域中, 单位时间内只有少量的分子能够与外 界环境接触释放 CO, 因此具有长时间缓释的能力. 在 $\mathrm{pH}=9$ 的条件下, $\mathrm{CO}$ 释放的半衰期约为 $60 \mathrm{~min}$, 而在 $\mathrm{pH}=4$ 的酸性条件下, $\mathrm{CO}$ 释放的半衰期缩短到 $30 \mathrm{~min}$. 由于 $\mathrm{P}(\mathrm{OEGA})-\mathrm{b}-\mathrm{P}(4 \mathrm{VP})$ 自身并不含有对 $\mathrm{pH}$ 敏感的化学 键, 因此其 $\mathrm{pH}$ 响应释放 $\mathrm{CO}$ 的现象可能来自于部分未 
反应的吡啶基团在酸性条件下质子化, 增加水分子进入 纳米胶束内部并与供体分子发生相互作用的能力. 这种 利用纳米材料表面亲疏水性变化而控制 $\mathrm{CO}$ 供体释放的 策略, 为构建 $\mathrm{pH}$ 响应型 $\mathrm{CO}$ 纳米控释材料提供了新的 思路(图 1).

(a)

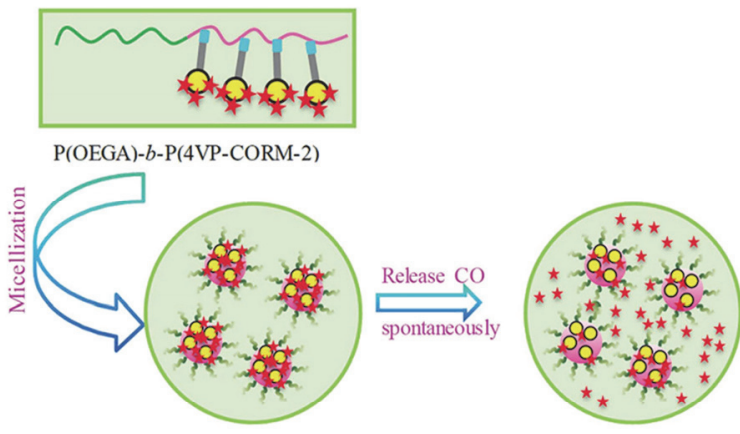

(b)

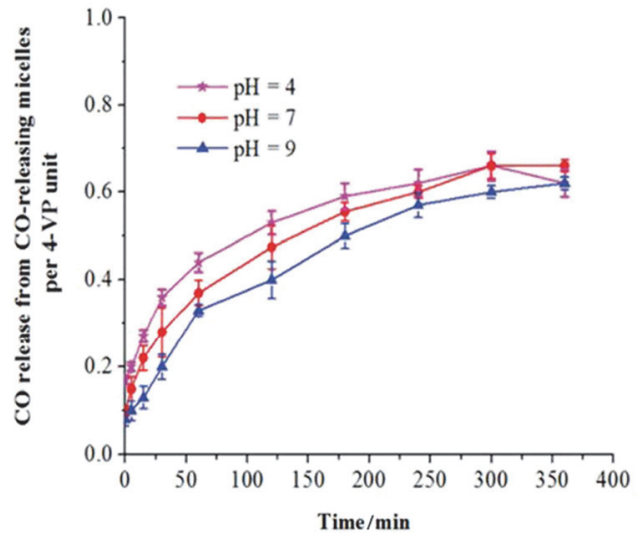

图 1 (a) 聚合物纳米胶束 P(OEGA)-b-(4VP-CORM-2)释放一氧化碳 的示意图; (b) 在不同 $\mathrm{pH}$ 值时, $\mathrm{CO}$ 的释放量随时间变化的曲线 ${ }^{[48]}$

Figure 1 (a) Schematic of the release of carbon monoxide from polymer nanomicelle $\mathrm{P}(\mathrm{OEGA})-\mathrm{b}-(4 \mathrm{VP}-\mathrm{CORM}-2)$; (b) The curve of CO release changing with time at different $\mathrm{pH}$ values ${ }^{[48]}$. Adopted with permission from ref. 48 Copyright 2016 WILEY-VCH Verlag GmbH \& Co. KGaA, Weinheim.

\section{$2.2 \mathrm{H}_{2} \mathrm{O}_{2}$}

过氧化氢 $\left(\mathrm{H}_{2} \mathrm{O}_{2}\right)$ 是需氧生物中普遍存在的代谢中间 产物 ${ }^{[49 \sim 52]}$. 一般情况下, 细胞内的 $\mathrm{H}_{2} \mathrm{O}_{2}$ 含量处于一个 较低水平 ${ }^{[53]}$. 但是据文献报道, 肿瘤细胞通常会产生大 量的 $\mathrm{H}_{2} \mathrm{O}_{2}$, 使得肿瘤微环境中的 $\mathrm{H}_{2} \mathrm{O}_{2}$ 水平显著提高 ${ }^{[50]}$. $\mathrm{H}_{2} \mathrm{O}_{2}$ 在生物体内并不稳定, 其分解后能够产生大量羟 基自由基等活性氧族(reactive oxygen species, ROS ${ }^{[53 \sim 55]}$. 这类物质具有较高的反应活性, 促使 CORMs 中的过渡 金属和羰基之间的化学键断裂, 加速 $\mathrm{CO}$ 释放. 因此, 研究者们设想利用肿瘤内高浓度的 $\mathrm{H}_{2} \mathrm{O}_{2}$ 作为天然的靶 点, 设计 $\mathrm{H}_{2} \mathrm{O}_{2}$ 响应型 $\mathrm{CO}$ 控释体系, 在肿瘤原位释放 $\mathrm{CO}$, 杀伤肿瘤细胞.

$\mathrm{He}$ 等 ${ }^{[56]}$ 利用中空介孔二氧化硅纳米颗粒负载羰基 锰, 构建了一种新颖的 $\mathrm{H}_{2} \mathrm{O}_{2}$ 响应型 $\mathrm{CO}$ 控释纳米材料 $\left(\mathrm{Mn}_{2}(\mathrm{CO})_{10} @ \mathrm{hMSN}\right)$. 在该体系中, $\mathrm{CO}$ 供体 $\mathrm{Mn}_{2}(\mathrm{CO})_{10}$
可以在羟基自由基的作用下分解释放 $\mathrm{CO}$, 同时生成锰 离子. 而锰离子通过类 Fenton 反应催化 $\mathrm{H}_{2} \mathrm{O}_{2}$ 生成更多 的羟基自由基，使得 $\mathrm{Mn}_{2}(\mathrm{CO})_{10}$ 加速分解，达到快速释 放 $\mathrm{CO}$ 的目的. 由于绝大部分肿瘤细胞中的 $\mathrm{H}_{2} \mathrm{O}_{2}$ 含量要 高于正常细胞, 这种 $\mathrm{H}_{2} \mathrm{O}_{2}$ 响应型 $\mathrm{CO}$ 控释纳米材料能够 实现选择性地释放 $\mathrm{CO}$, 利用其毒性作用靶向杀伤肿瘤 细胞. 除了无机硅材料之外, 有机金属框架材料具有极 大的比表面积、可调的孔道和易修饰等特点，也可以用 于负载 $\mathrm{CO}$ 供体. $\mathrm{He}$ 等 ${ }^{[57]}$ 利用含钛的金属有机框架材料 (Ti-MOF)和 CO 供体的配位作用，构建了新颖的 CO 控 释纳米材料(MnCO@Ti-MOF). 在 $\mathrm{H}_{2} \mathrm{O}_{2}$ 存在的条件下, $\mathrm{MnCO} @ \mathrm{Ti}-\mathrm{MOF}$ 可以快速释放 CO 分子, 并且其反应副 产物还具有蓝色菼光, 可以满足在细胞内实时监控 $\mathrm{CO}$ 释放过程的需求(图 2).

除了利用细胞内自有的 $\mathrm{H}_{2} \mathrm{O}_{2}$ 外, 通过外界刺激也 可以诱导细胞内 $\mathrm{H}_{2} \mathrm{O}_{2}$ 的含量升高, 促使 $\mathrm{H}_{2} \mathrm{O}_{2}$ 响应型的 $\mathrm{CORMs}$ 释放 $\mathrm{CO}$, 从而实现 $\mathrm{CO}$ 的可控释放. $\mathrm{Gu}$ 等 ${ }^{[58]}$ 以 第 3 代聚 $(L$-赖氨酸)树突状大分子为核, 通过负载光敏 剂分子 Ce6 和 CO 供体 CORM-401, 制备了一种能够协 同光动力治疗和具有 $\mathrm{CO}$ 控释能力的纳米材料 (CORM@G3DSP-Ce6). 在近红外光照射下, Ce6 分子可 以在细胞内产生大量的 $\mathrm{ROS}$, 提高细胞内 $\mathrm{H}_{2} \mathrm{O}_{2}$ 的含量. 与此同时, CORM-401 在高 $\mathrm{H}_{2} \mathrm{O}_{2}$ 的条件下可以快速释放 $\mathrm{CO}$. 体外和体内实验的结果表明, 光动力治疗和 $\mathrm{CO}$ 之 间存在良好的协同效应. 因此，这种以纳米材料为基础 的 $\mathrm{CO}$ 供体构建策略不仅可以实现 $\mathrm{CO}$ 的可控释放，还 能将 CO 与肿瘤治疗相结合，扩展其应用领域.

\section{3 光}

光作为一种外界刺激源, 由于其具有非侵入性、廉 价性和可操作性等特点而受到广泛的关注 ${ }^{[30,59]}$. 在现有 的 $\mathrm{CO}$ 供体中, 许多以 $\mathrm{Mn} 、 \mathrm{Fe} 、 \mathrm{~W}$ 原子为配位中心的 羰基化合物及其衍生物都具有光响应释放 $\mathrm{CO}$ 的能力, 例如 $\left[\mathrm{Mn}(\mathrm{CO})_{3}(\mathrm{tpm})\right] \mathrm{PF}_{6}$ 、 $\left[\mathrm{Fe}(\mathrm{CO})\left(\mathrm{N}_{4} \mathrm{Py}\right)\right]\left(\mathrm{ClO}_{4}\right)_{2}$ 、 $\mathrm{Na}_{3}\left[\mathrm{~W}(\mathrm{CO})_{5}\right.$ (TPPTS)]等 ${ }^{[14,60 \sim 63]}$. 将这些光敏感型 $\mathrm{CO}$ 供体与纳米材料相结合, 利用纳米材料独特的物理化学 性质，能够改善这些供体的生物相容性，或大大提高它 们在生物体内的稳定性.

\subsection{1 紫外-可见光}

由于紫外光的光子能量较高, 可以打断光敏感或不 稳定的化学键, 触发 $\mathrm{CO}$ 释放. 因此, 大多数光响应型 $\mathrm{CO}$ 供体都是紫外光或蓝紫光敏感的. Berreau 等 ${ }^{[64]}$ 报道 了一种不含金属的有机光敏 CO 供体前药, 在可见光 ( 460 nm) 的照射下可控地释放 CO. 这种基于 3-羟基 苯的喹诺酮框架的前药分子，在与白蛋白和硫醇反应后 具有可见光响应控释 $\mathrm{CO}$ 的能力. 实验结果显示, 这种 CO 递送系统既能够有效地杀伤肿瘤细胞也可产生显著 
(a)

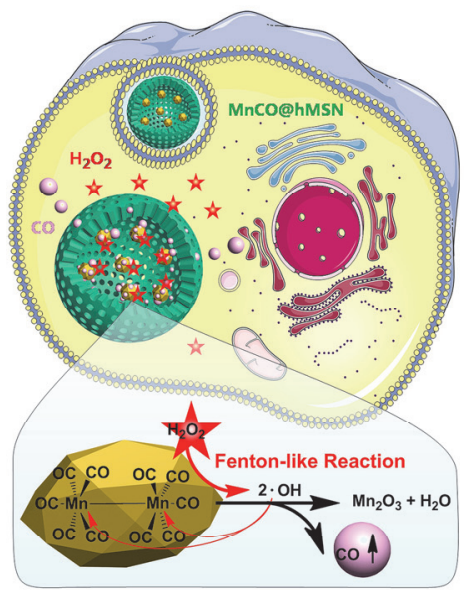

(b)

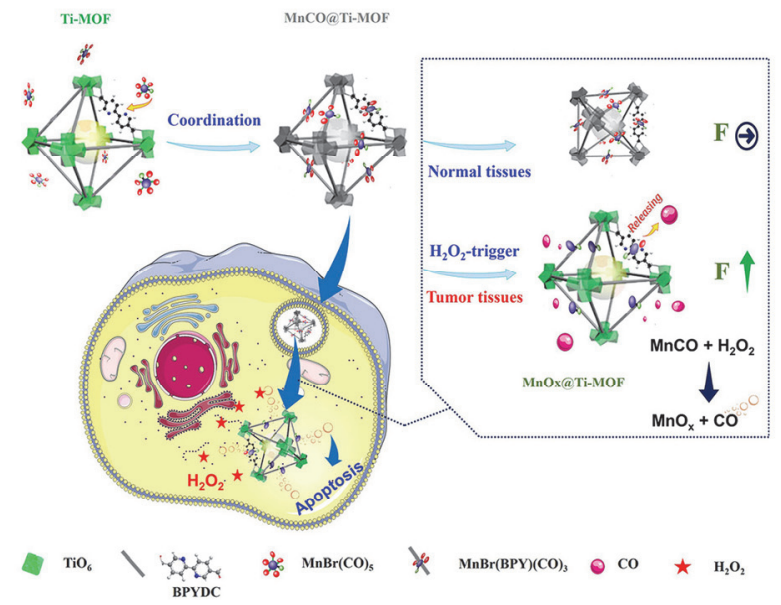

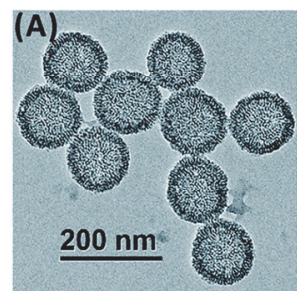
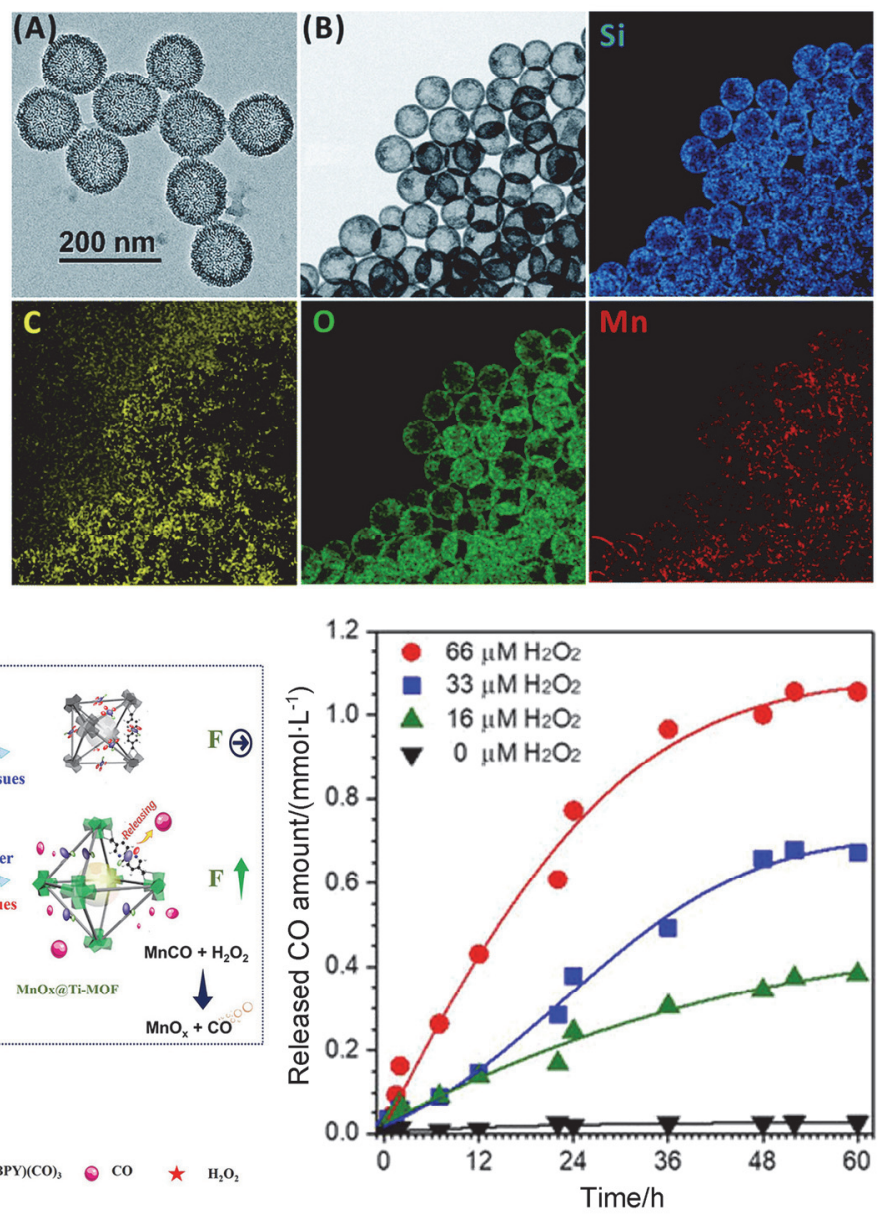

图 2 (a) MnCO@hMSN 纳米颗粒的结构和工作机理(左); hMSN 载体的 TEM 图像(A), 以及 MnCO@hMSN 纳米医学的 TEM 图像(B)和相应的元 素映射(右) ${ }^{[56]}$; (b) $\mathrm{MnCO} @ \mathrm{Ti}-\mathrm{MOF}$ 的结构及其在 $\mathrm{H}_{2} \mathrm{O}_{2}$ 作用下释放 $\mathrm{CO}$ 机理的示意图(左); 不同浓度 $\mathrm{H}_{2} \mathrm{O}_{2}$ 处理 $\mathrm{MnCO} @ \mathrm{Ti}-\mathrm{MOF}$ 时释放 CO 的实时 浓度曲线(右) $)^{[57]}$

Figure 2 (a) Structure and working mechanism of MnCO@hMSN nanoparticles (left); TEM image of the hMSN carrier (A), and TEM image and corresponding element mapping of the MnCO@hMSN nanomedicine (B) (right $)^{[56]}$. Reproduced from Ref. 56 with permission from The Royal Society of Chemistry 2017. (b) Schematic diagram of the structure and its mechanism of releasing CO of MnCO@Ti-MOF (left); Real-time concentration curve for releasing $\mathrm{CO}$ of MnCO@Ti-MOF at different concentrations of $\mathrm{H}_{2} \mathrm{O}_{2}$ (right $)^{[57]}$. Adopted with permission from ref. 57 Copyright $2018 \mathrm{Wiley-VCH} \mathrm{Verlag}$ GmbH\& Co. KGaA, Weinheim

的抗炎作用. 相对于其他的 $\mathrm{CO}$ 供体分子，该前药分子 在体内能迅速与白蛋白结合, 形成较为稳定的纳米颗 粒, 具有靶向输运的潜力. 近年来, 由蛋白质分子自组 装形成的中空笼形蛋白, 由于具有结构可塑性和生物相 容性好的特点, 被广泛用于纳米颗粒的修饰与包装、药 物输运和靶向释放等领域. Ueno 等 ${ }^{[65]}$ 将铁蛋白与 CO 供 体 $\mathrm{Mn}(\mathrm{CO})_{5} \mathrm{Br}$ 结合, 制备出一种蛋白修饰的光敏型 $\mathrm{CO}$ 纳米供体(MnCO-apo-R52C-rHLFr). 体外实验表明, 该 纳米材料在可见光 $(456 \mathrm{~nm})$ 照射下能够有效地释放 $\mathrm{CO}$, 并且其释放剂量可以受到光照时间和强度的控制. 随后 他们利用光控释放的 $\mathrm{CO}$ 激活哺乳动物细胞 NF- $\kappa \mathrm{B}$ 通 路, 展现出其在光控调节信号通路方面应用的潜力.

Schatzschneider 等 ${ }^{[66]}$ 通过铜催化的叠氮端炔环加成 反应将 $\left[\mathrm{Mn}(\mathrm{CO})_{3}(\mathrm{tpm})\right]^{+}$修饰到纳米二氧化硅表面, 构 建出具有光响应能力的 $\mathrm{CO}$ 输运纳米材料. 在 $365 \mathrm{~nm}$ 光
源的照射下，该纳米材料可以有效地响应光刺激，释放 $\mathrm{CO}$ 分子. 在硅纳米颗粒中, 介孔氧化硅具有比表面积 大、稳定性好的特点, 常被用于药物输运领域. Mascharak 等 ${ }^{[60]}$ 通过物理吸附的方法, 将光敏型的 $\mathrm{CO}$ 供体 $\left[\mathrm{Mn}(\mathrm{pqa})(\mathrm{CO})_{3}\right] \mathrm{ClO}_{4}$ 负载到介孔硅分子篎材料上，制备 了光响应型 CO 输运材料(Mn-CO@Al-MCM-41). 实验 结果显示, 该纳米材料即使在低强度的可见光照射下 $(10 \sim 50 \mathrm{~mW})$ 也具有较好的 CO 响应控释能力. 最重要 的是, 利用介孔硅材料可以将 CORMs 分解后的副产物 留在孔道中, 一定程度上避免了其产生的副作用. 为了 追踪 $\mathrm{CO}$ 输运材料和监控 $\mathrm{CO}$ 在细胞内的释放过程, Maldonado 等 ${ }^{[67]}$ 合成了一种能发射红色苂光的光敏型 $\mathrm{CO}$ 供体 fac- $\left[\mathrm{Re}(\mathrm{CO})_{3}(\mathrm{pbt})\left(\mathrm{PPh}_{3}\right)\right]\left(\mathrm{CF}_{3} \mathrm{SO}_{3}\right)$, 并将其负载 到介孔硅分子篎上, 得到了一种新颖的光响应型 $\mathrm{CO}$ 输 运材料. 在该体系中, $\mathrm{CO}$ 供体在蓝紫光照射下能发射红 
色荧光, 且在释放 $\mathrm{CO}$ 后由于分子结构变化而导致荧光 淬灭. 因此, 利用这种 Re-CO@Al-MCM-41 纳米颗粒可 以在细胞内准确定位材料位置和实时定量地监控 $\mathrm{CO}$ 释 放过程, 具有重要的应用价值.

除氧化硅纳米颗粒 ${ }^{[59,68,69]}$ 外, 碳纳米材料、有机金 属框架材料等新型纳米材料也可以作为载体, 构建 $\mathrm{CO}$ 可控输运体系. Furukawa 等 ${ }^{[70]}$ 将 $\mathrm{CO}$ 供体 $(\mathrm{MnBr}-$ $\left.(\mathrm{dmbpy})(\mathrm{CO})_{3}\right)$ 通过配位作用负载在有机金属框架材料 (UiO-67-bpy)表面, 制备了光敏型 $\mathrm{CO}$ 控释纳米材料 (CORF-1) (图 3b 左). 实验结果显示, CORF-1 在 $460 \mathrm{~nm}$ 光照条件下可以快速释放 $\mathrm{CO}$ (图 $3 \mathrm{~b}$ 右), 而单独的 $\mathrm{MnBr}(\mathrm{dmbpy})(\mathrm{CO})_{3}$ 在同样条件下几乎不能释放 $\mathrm{CO}$ 分 子. 进一步的研究发现, MOFs 材料为光敏型 CO 供体分 子提供了足够的空间位点, 可以有效抑制其聚集诱导淬 灭的现象, 从而提高光敏分子的光吸收效率. 因此, MOFs 材料在光响应型 CO 输运材料中的构建及其生物 医学应用中具有很大的优势.

\subsection{2 近红外光}

虽然紫外光和蓝紫光的光化学反应活性较高, 但在 实际临床应用中, 面临着组织穿透深度受限和光损伤的 问题，严重限制了光响应性气体释放纳米材料的应用. 如果将光响应的控制信号调整到近红外区, 利用近红外 光(NIR) 在生物组织内穿透深度大的特点, 可以扩展体 内释放 CO 的应用范围. Tang 等 ${ }^{[23]}$ 报道了一种近红外响 应的 $\mathrm{CO}$ 供体分子, 该分子首先与 $\mathrm{H}_{2} \mathrm{O}_{2}$ 反应形成具有光 活性的供体, 然后可以在 $808 \mathrm{~nm}$ 近红外光照射下释放 $\mathrm{CO}$, 具有 $\mathrm{H}_{2} \mathrm{O}_{2}$ 和近红外光双重响应性. 随着纳米技术 的发展, 一些利用具有近红外响应能力的纳米材料, 可 以作为载体输运 $\mathrm{CO}$, 具有很好的生物应用前景. Ford 等 ${ }^{[71]}$ 将稀土上转换纳米材料与光敏型 CORMs 结合, 制 备出近红外光响应型 $\mathrm{CO}$ 控释材料. 在近红外光的照射 下, 上转换纳米材料可以吸收近红外光子并发射出可见 光或蓝紫光, 将能量传递给吸附在其表面的光敏 $\mathrm{CORMs}$, 促使 CO 释放. 上转换纳米材料的荧光可以通 过不同元素掺杂的方法调控, 因此可以匹配多种不同波 长敏感的 CORMs, 增加其在应用中的灵活性. 除了基 于稀土元素的上转换纳米材料之外, 基于三线态-三线 态湮灭(TTA)机理的分子或纳米材料也可以有效利用长 波长的激发光. Schiller 等 ${ }^{[72]}$ 发展了一种利用红光 635 $\mathrm{nm}$ )激活光敏性 $\mathrm{CO}$ 释放分子 $\mathrm{Mn}_{2}(\mathrm{CO})_{10}$ 的策略. 他们利 用四苯基四苯并卟啉钯作为三线态淬灭的敏化剂, 吸收 $635 \mathrm{~nm}$ 的近红外光, 发生 TTA 后将能量传递给紫外光 敏感的 CO 供体, 以此释放 CO. 除了这些荧光材料外, 具有 NIR 吸收能力的氧化石墨烯(GO)纳米片也可以作 为功能性载体, 构建近红外光响应型 $\mathrm{CO}$ 输运体系. Chen 等 ${ }^{[73]}$ 设计合成了一种新型的石墨烯-羰基锰复合 纳米材料, 实现了近红外区的 $\mathrm{CO}$ 气体可控释放(图 3c). 这种新型的纳米药物利用 GO 的 NIR 吸收效应和光电转 (a)

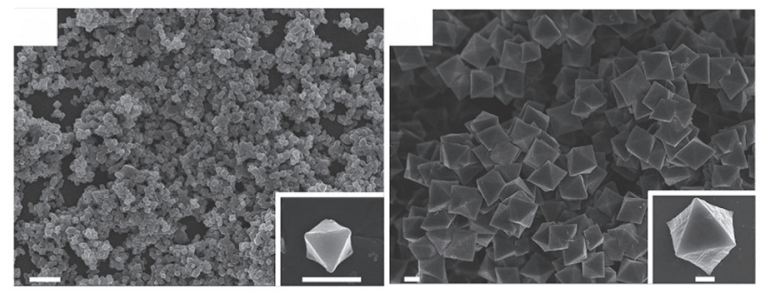

(b)

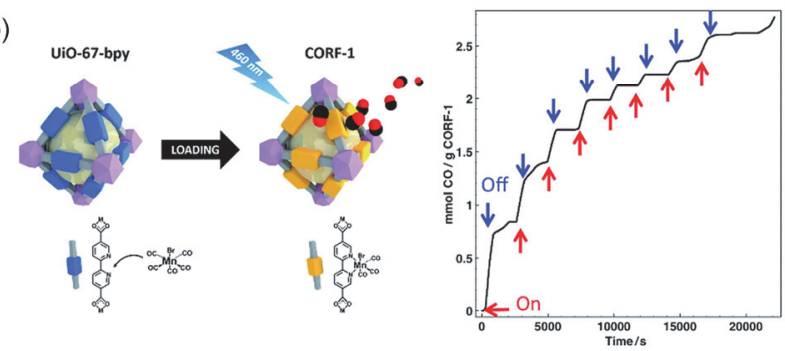

(c)

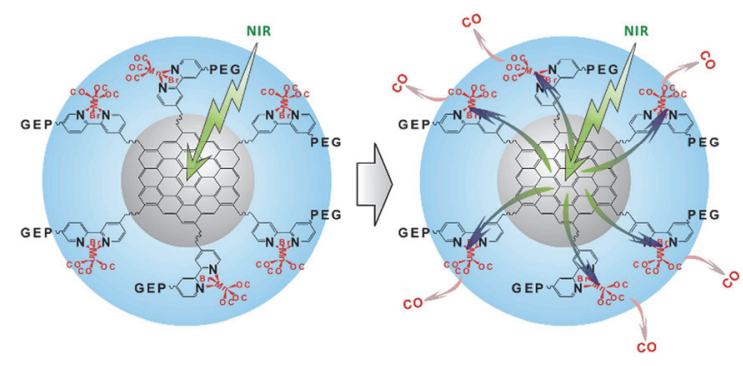

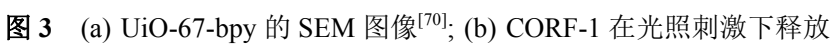
$\mathrm{CO}$ 的示意图(左); CORF-1 的光响应释放 $\mathrm{CO}$ 的开关性能(右) ${ }^{[70]}$; (c) PEG-BPY $\left[\mathrm{MnBr}(\mathrm{CO})_{3}\right]-\mathrm{GO}$ 的分子结构和 $\mathrm{CO}$ 释放机制示意图 ${ }^{[73]}$

Figure 3 (a) SEM image of Uio-67-bpy ${ }^{[70]}$; (b) Schematic diagram of CORF-1 releasing CO under light stimulation (left); CORF-1 light response CO release switch performance (right) ${ }^{[70]}$. Reproduced from Ref. 70 with permission from The Royal Society of Chemistry 2017. (c) Molecular structure and $\mathrm{CO}$ release mechanism of PEG-BPY $\left.\mathrm{MnBr}(\mathrm{CO})_{3}\right]-$ $\mathrm{GO}^{[73]}$. Adopted with permission from ref. 73 Copyright 2015 WILEY$\mathrm{VCH}$ Verlag $\mathrm{GmbH} \& \mathrm{Co}$. KGaA, Weinheim

化效应，将吸收的 NIR 光转化为活性电子，用于激活连 接在 GO 上的羰基锰释放 $\mathrm{CO}$ 气体. Kong 等 ${ }^{[74]}$ 系统研究 了羰基钌团簇( $\mathrm{Ru}-\mathrm{CO})$ 在氧化石墨烯上的吸附特性，并 以此设计了一种近红外光控释 $\mathrm{CO}$ 的纳米材料. 该种基 于石墨烯纳米材料的 $\mathrm{CO}$ 控释体系具有较高的药物负载 率和“按需释药”的特性, 在细胞内能够可控地释放 $\mathrm{CO}$, 并且展现出较高的生物学活性.

虽然上转换纳米材料 ${ }^{[75 ~ 77]}$ 和石 墨烯纳米材料 ${ }^{[78]}$ 可 以吸收 NIR 光, 并将其能量传递给光敏型 CORMs, 但 是上转换过程和光电转换的效率较低, 对近红外光的利 用程度不高, 影响 CO 的释放效率. Yeh 等 ${ }^{[79]}$ 利用介孔普 鲁士蓝纳米颗粒，通过共价修饰的方法负载 $\mathrm{CO}$ 供体羰 基铁，制备了一种近红外光响应型 $\mathrm{CO}$ 控释纳米材料 (m-PB-CO). 普鲁士蓝纳米颗粒具有较高的光热转换效 率, 在近红外光照射条件下, 能够促使周围环境温度快 速升高, 加速 $\mathrm{CO}$ 供体分解释放 CO. 随后, 他们将聚乙 二醇修饰的 m-PB-CO 纳米材料用于肿瘤光热治疗中, 发现 CO 与光热治疗有明显的协同效应. 因此, 
m-PB-CO 纳米材料可以同时实现近红外光响应的 $\mathrm{CO}$ 可控释放和 $\mathrm{CO}$ 增敏的光热治疗, 为光响应型 $\mathrm{CO}$ 控释 纳米材料在生物治疗领域的应用打下了基础.

\section{4 磁场}

相对于光而言, 磁场在生物体内具有更高的穿透深 度, 且副作用相对较小 ${ }^{[28,80,81]}$. 因此, 磁场可以作为一 种理想的外源性刺激用于构建响应型 CO 控释体系. 以 氧化铁为代表的磁性纳米材料, 具有独特的磁响应能力 和简单可控的制备方法, 已经被广泛应用于生物治疗和 医学诊断领域 ${ }^{[82]}$. Janiak 等 ${ }^{[83]}$ 将含钌的一氧化碳供体 $\mathrm{Ru}_{2} \mathrm{Cl}_{4}(\mathrm{CO})_{6}$ 修饰到氧化铁纳米颗粒表面得到了一种磁 控 CO 输运材料(CORM@IONP), 首次在交变磁场作用 下实现了 $\mathrm{CO}$ 的可控释放. 为了提高 $\mathrm{CO}$ 控释纳米材料 在生理溶液中的分散性和稳定性, 他们又在 CORM@IONP 表面修饰了一层葡聚糖. 氧化铁纳米颗 粒在交变磁场作用下可以产生大量的热, 促使 CORM 分子分解释放 CO. 实验结果显示, 该纳米复合物在 $20{ }^{\circ} \mathrm{C}$ 水溶液中相对稳定, 其 $\mathrm{CO}$ 释放的半衰期接近 900 $\mathrm{min}$, 而在交变磁场作用下, $\mathrm{CO}$ 释放的半衰期约为 155 $\min$. 而在生理环境中 $\left(37^{\circ} \mathrm{C}\right), \mathrm{CO}$ 释放的半衰期分别为 $172 \mathrm{~min}$ (无磁场)和 $65 \mathrm{~min}$ (有磁场). 由于钉配位的 CORM 存在 CO 缓释的现象, Janiak 等 ${ }^{[84]}$ 提出了利用相 对稳定的肟类 CORM 作为供体, 通过共价作用修饰在 IONP 表面, 制备了一种长效稳定且具有磁控制释放功 能的 CO 输运纳米材料(Dextran@oxime-CORM@IONP). 这种纳米材料稳定性和生物安全性较好, 在浓度 100 $\mu \mathrm{g} \cdot \mathrm{mL}^{-1}$ 的条件下也没有任何细胞毒性, 在生物应用领 域有着巨大的潜力.

\section{3 一氧化碳(CO)控释纳米材料的生物医学应 用}

近年来的研究发现, 一氧化碳作为一种气体信使分 子, 可以调控一系列的生理活动 ${ }^{[8,85 \sim 88]}$. 但是现有的一 氧化碳供体由于缺少靶向性、释放速率受外界影响太大 等问题，使得这类供体在临床上的使用受到了限制，为 了解决这些问题, 国内外学者借鉴纳米材料 ${ }^{[89]}$ 用于药 物输运的研究思路, 运用纳米材料作为现有一氧化碳供 体的载体进行了初步的探索, 并取得了很多重要的结论 和有意义的成果. 借助纳米材料的性能优势能够提高 $\mathrm{CO}$ 释放的靶向性, 改善释放性能, 有利于促进 $\mathrm{CO}$ 控释 纳米材料在炎症 ${ }^{[00,91]}$ 、抗菌 ${ }^{[92]}$ 以及癌症 ${ }^{[31,37]}$ 等生物医学 领域的应用.

\section{1 一氧化碳(CO)控释纳米材料在炎症治疗领域的应 用}

近年来, 研究者们发现一氧化碳(CO) 和 $\mathrm{CO}$ 供体分 子(CORMs) 在调节免疫抑制和抗炎症方面有着广泛的 应用 ${ }^{[72,91,93 ~ 97]}$. 一氧化碳参与调节丝裂原活化蛋白激酶 (mitogen-activated protein kinase, MAPK)相关信号通路 和 c-Jun 氨基末端激酶(c-Jun N-terminal kinases, JNK)信 号通路，抑制肿瘤坏死因子- $\alpha$ 、巨噬细胞炎性蛋白因 子-1 $\beta$ 等炎症细胞因子的释放 ${ }^{[98,99]}$. 此外, 在 Jurkat $\mathrm{T}$ 细 胞中, 由 HO-1 产生的 CO 还被证明能够通过下调 IL-2 分泌的途径抑制 $\mathrm{T}$ 细胞的增殖 ${ }^{[100]}$. 多项体外实验证实 CO 供体分子, 例如 CORM-2 和 CORM-3, 可通过增强 内源性 HO 的活性和 HO-1 的表达抑制 LPS 介导的 RAW264.7 小鼠巨噬细胞炎症反应 ${ }^{[21,96,101]}$. CORM-3 能 够抑制凝血酶、细胞因子及缺氧所诱导的 BV-2 小胶质 细胞炎症反应，减少一氧化氮和 TNF- $\alpha$ 的产生 ${ }^{[90,91,102]}$. 此外, 有研究显示在抑制 MAPK 通路的过程中, CORM-3 的抗炎作用明显增强, 可见 CORM-3 的抗炎机 制不仅仅是单纯地抑制酶的活性，而是与更深层次的细 胞信号通路密切相关 ${ }^{[103,104]}$.

Hubbell 等 ${ }^{[105]}$ 合成了一种由含有 $\mathrm{CO}$ 供体的两亲性 嵌段共聚物自组装形成的聚合物纳米胶束(CO-releasing micelle). 这种纳米胶束可以在放基的刺激下释放 $\mathrm{CO}$, 其释放速率和释放浓度与 $\mathrm{CO}$ 供体的类型和颈基物质含 量有关. 随后, 他们在体外模型中利用稳定转染 SEAP 报告基因的 THP-1 Blue 细胞验证了该纳米胶束的抗炎 症效应. LPS 可以刺激激活 NF- $\mathrm{KB}$ 信号通路, 使得 THP-1 Blue 细胞分泌 SEAP. 将细胞与 CO-releasing micelle 共同孵育可以大大降低 SEAP 的释放量, 说明这 种纳米胶束能够有效抑制 LPS 诱导的炎症反应. 但是, 单独的 $\mathrm{CO}$ 供体 $\mathrm{Ru}(\mathrm{CO}){ }_{3} \mathrm{Cl}$ (glycinate)并没有表现出抑制 效应, 反而加剧了炎症反应的程度. 他们推测这可能与 含 $\mathrm{Ru}$ 的副产物有关. 细胞毒性实验显示, $\mathrm{CO}$ 供体 $\mathrm{Ru}(\mathrm{CO})_{3} \mathrm{Cl}$ (glycinate) 在较高浓度下有一定的细胞毒性 $\left(\mathrm{IC}_{50}=600 \mu \mathrm{mol} / \mathrm{L}\right)$, 而 CO-releasing micelle 在 $1 \mathrm{mmol} / \mathrm{L}$ 浓度也没有明显的细胞毒性. 此外, Hasegawa 等 ${ }^{[106]}$ 利 用含苯基硼酸的纳米粒子 (PBANPs) 负载 $\mathrm{CO}$ 供体 $\mathrm{CO}-\mathrm{DOPA}$ ，制备了一种可释放 $\mathrm{CO}$ 气体的聚合物纳米颗 粒(CONPs), 将其用于抗炎症治疗. 在 LPS 诱导的炎症 模型中, CONPs、CO 供体 CO-DOPA 和 CORM-3 都能 显著抑制巨噬细胞分泌白介素 IL-6. 虽然 CONPs 的抗 炎症效果要稍差于 CO 供体 CO-DOPA 和 CORM-3, 但 其细胞毒性要远小于后两者. 由此可见，通过采用纳米 胶束负载 CORMs 的策略可以大大提高 CO 控释材料的 生物安全性, 为一氧化碳在炎症治疗领域的应用提供了 一个安全的输运平台.

除了可以降低毒性之外, 利用纳米载体负载 CORMs 还能够改变 $\mathrm{CO}$ 的释放速度, 从而对其生物学 效应产生一定的影响. Kim 等 ${ }^{[107]}$ 以甘油三月桂酸酯为 核心, 以 Tween 20、Span 20 和 Myrj S40 为表面活性剂, 并将 CO 供体 CORM-2 吸附在内层形成了一种可释放 $\mathrm{CO}$ 的脂质纳米颗粒(CORM-2-NPs). 在 LPS 诱导的体外 炎症模型中, CORM-2-NPs 表现出更好的抗炎症能力. 在体内实验中, CORM-2-NPs 和 CORM-2 都可以有效抑 
制卡拉胶引发的炎症而导致的大鼠爪部水肿现象，且抗 炎症效果要好于目前临床上常用的非甾体抗炎药-吲哚 美辛. 和体外实验相似的是, CORM-2-NPs 的抗炎症效 果要好于单独的 CORM-2. 通过检测 $\mathrm{CO}$ 的释放曲线, 他们发现 CORM-2 在溶液中快速释放 CO, 在 $10 \mathrm{~min}$ 时 的释放量可达 $70 \%$; 而 CORM-2-NPs 可以持续数小时稳 定地释放 CO. 因此, 在体内和体外的炎症模型中, CORM-2-NPs 都表现出更好的抗炎症效果(图 4). Ueno 等 ${ }^{[108]}$ 设计了一种负载 CORMs 铁蛋白笼 $(\mathrm{Fr})$ 体系 Fr-CORM，这种蛋白笼结构能够降低 $\mathrm{CO}$ 释放的速率， 延长 $\mathrm{CO}$ 供体释放的半衰期, 因而能够长时间持续保持 生物学活性. 实验结果显示, 该体系的 $\mathrm{CO}$ 释放速率比 CORM-3 要慢 18 倍以上, 其激活核转录因子 NF- $\mathrm{NB}$ 的 效率也要高出 10 倍, 有显著的抗炎症作用. 此后, Ueno 等 ${ }^{[109]}$ 又进一步发展了一种光敏型 Fr-CORM 体系, 在 HEK293 细胞中可控地激活了 NF- $\mathrm{BB}$ 信号通路. 这种基 于铁蛋白笼结构的 $\mathrm{CO}$ 控释纳米材料具有生物安全性 好、细胞摄取量大、长时间缓释等特点, 是一种性能良 好的 CO 递送体系. 综上所述, CO 控释纳米材料具有低 毒性和长效释放两大优势, 在炎症治疗领域具有良好的 应用前景.

(a)
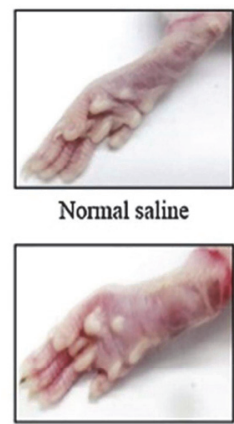

CORM-2 solution

(b)

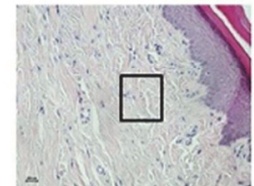

Normal saline

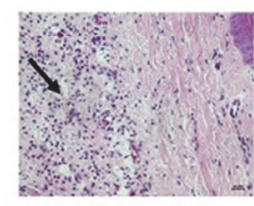

CORM-2 solution

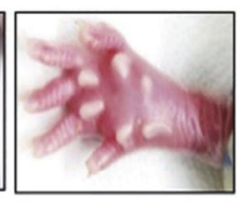

Carrageenan

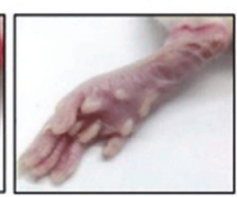

CORM-2-NPS

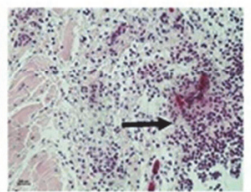

Carrageenan

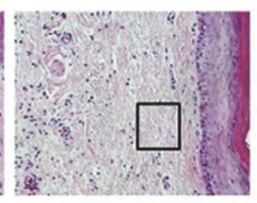

CORM-2-NPs

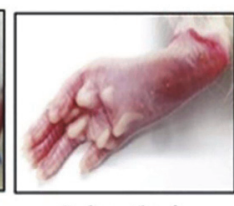

Indomethacin
图 4 (a) 大鼠注射卡拉胶诱导炎症之后，足部的实时成像图片; (b) 卡拉胶诱导后, 大鼠足部组织的病理变化和 H\&E 染色图片 ${ }^{[107]}$

Figure 4 (a) Real-time imaging of the rats' feet after the injection of carrageenan-induced inflammation; (b) Microscopic images of H\&E staining sections of carrageenan-induced rat foot tissues ${ }^{[107]}$. Reproduced from Ref. 107 with permission from 2016 European Journal of Pharmaceutics and Biopharmaceutics

\section{2 一氧化碳(CO)控释纳米材料在抗菌治疗领域的应 用}

目前, 病原体的耐药性导致抗生素疗效下降的问题 对全球公共卫生造成很大的威胁，这也为寻求新的抗菌

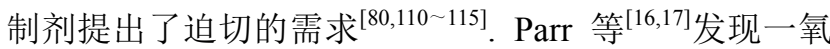
化碳 $(\mathrm{CO})$ 是铜绿假单胞菌呼吸链的有效抑制剂. 此外, CO 能通过 P38 介导的 TLR4 表达促进巨噬细胞对大肠 杆菌的吞噬, 并且还能作为小鼠微生物败血症宿主防御 反应的重要介质 ${ }^{[114,116,117]}$. 因此, CO 有望在抗菌领域发 挥巨大的作用. 然而在实际应用中, 使用 $\mathrm{CO}$ 气体作为 抗菌剂主要存在剂量难以控制而导致毒副作用的问题. 而 CORMs 可以在特定条件释放 CO, 其剂量相对容易 控制. Saraiva 等 ${ }^{[110]}$ 发现 CO 供体分子(CORM-2 和 CORM-3)对多种细菌有明显的抑制效应. 在溶液中添 加 CORM-2 或者 CORM-3 会导致大肠杆菌和金黄色葡 萄球菌在 $30 \mathrm{~min}$ 内存活率下降, $4 \mathrm{~h}$ 后无法继续生长. Persson 等 ${ }^{[118]}$ 报道了 CORM-2 可以抑制具有多重耐药性 的 UPEC 大肠细菌, 具有良好的临床应用前景. 此外, 其它多种 CORMs 也被报道能够对抗耐抗生素的致病 菌 ${ }^{[119,120]}$. 但是, CORMs 仍然存在稳定性差、难溶于水 等缺点. 因此, 研究者们尝试利用纳米材料的优势解决 这些问题.

聚合物纳米载体具有药物负载量大、毒副作用小、 免疫原性低的特点, 被广泛应用于药物输运领域 ${ }^{[24,121]}$. Boyer 等 ${ }^{[92]}$ 合成了一系列聚合物纳米载体, 将其与 $\mathrm{CO}$ 供体结合得到多种可以释放 $\mathrm{CO}$ 的聚合物纳米颗粒. 他 们发现这种具有 $\mathrm{CO}$ 缓释能力的聚合物纳米颗粒与 CORM-2 一样, 能够对铜绿假单胞菌产生很强的抑制作 用. 实验结果显示, $\mathrm{CO}$ 缓释纳米颗粒对悬浮的或生物膜 中的细菌都有明显的杀伤效应，其效果要好于单独的 CORM-2. 并且, $\mathrm{CO}$ 缓释聚合物纳米颗粒的毒性要远小 于 CORM-2, 其稳定性和水溶性也得到大幅提升. 因此, 这种性能优异且安全无毒的 CO 缓释聚合物纳米材料在 抗菌领域具有广阔的应用前景.

\section{3 一氧化碳(CO)控释纳米材料在癌症治疗领域的应 用}

在较早的时候, 研究人员就发现 $\mathrm{CO}$ 气体对肿瘤细 胞有促调亡的作用 ${ }^{[55,122 ~ 124]}$. 然而, $\mathrm{CO}$ 气体或者大部分 CORMs 存在靶向性差、剂量不可控和潜在毒性等问题, 限制了其在肿瘤治疗领域的应用. 由于纳米颗粒 ${ }^{[125]}$ 在 肿瘤部位存在明显的渗透滞留增强效应(Enhanced permeability and retention effect, EPR), 尺寸合适的纳米颗 粒载体可以选择性地富集到肿瘤组织, 实现特定靶向的 药物输运 ${ }^{[126]}$. 因此, 运用纳米材料作为药物载体负载 一氧化碳供体(CORMs), 能够提高靶向性和改进释放性 能, 已然成为当前的研究热点. 
$\mathrm{He}$ 等 ${ }^{[56]}$ 基于多孔氧化硅纳米颗粒构建了 $\mathrm{H}_{2} \mathrm{O}_{2}$ 响应 的 $\mathrm{CO}$ 释放纳米材料 $(\mathrm{MnCO} @ \mathrm{hMSN})$. 这种纳米材料释 放 $\mathrm{CO}$ 的速率与 $\mathrm{H}_{2} \mathrm{O}_{2}$ 的浓度呈正相关, 而肿瘤细胞中的 $\mathrm{H}_{2} \mathrm{O}_{2}$ 水平较高, 因此 $\mathrm{MnCO} @ \mathrm{hMSN}$ 可以在肿瘤细胞内 快速释放 $\mathrm{CO}$, 杀伤肿瘤细胞. 这种利用肿瘤细胞和正 常细胞内含物的浓度差异而实现靶向杀伤的策略, 对于 提高治疗效果、降低毒副作用具有较为重要的科学意义. 在此基础上, $\mathrm{Gu}$ 等 ${ }^{[58]}$ 利用同时负载光敏剂分子 $\mathrm{Ce} 6$ 和 CO 供体 CORM-401 的纳米材料(CORM@G3DSP-Ce6), 通过光动力治疗的形式在肿瘤细胞内产生大量的 ROS, 提高细胞内 $\mathrm{H}_{2} \mathrm{O}_{2}$ 的含量. 高浓度的 $\mathrm{H}_{2} \mathrm{O}_{2}$ 可以促进 CORM-401 快速释放 CO, 达到肿瘤治疗的目的(图 5).

除了利用 $\mathrm{CO}$ 的毒性直接杀伤肿瘤细胞外, 将 $\mathrm{CO}$ 与其它治疗方式进行联合, 不仅可以增强治疗效果, 还
可以降低单一治疗的剂量以减轻毒副作用. Yeh 等 ${ }^{[79]}$ 将 $\mathrm{CO}$ 供体羰基铁负载到 PEG 修饰的普鲁士蓝纳米颗粒表 面(m-PB-CO), 在近红外光的照射下, 实现可控释放 $\mathrm{CO}$, 并与光热治疗进行协同进行肿瘤治疗. 活体实验 结果显示, 在修饰 PEG 后的 m-PB-CO 与 $0.8 \mathrm{~W} / \mathrm{cm}^{2}$ 激 光照射的治疗组中，小鼠皮下移植瘤几乎完全消失. 并 且在治疗过程中小鼠的血氧饱和度保持稳定，没有任何 急性毒性现象的发生. 除了与光热治疗协同之外, CO 与 化疗等传统肿瘤治疗方法也有协同作用(图 6). Zhang 等 ${ }^{[127]}$ 将碳点和 $\mathrm{Ag}_{3} \mathrm{PO}_{4}$ 纳米粒子掺杂到 $\mathrm{C}_{3} \mathrm{~N}_{4}$ 中，制备一 种光催化纳米材料 $\mathrm{AgCCN}$, 将 $\mathrm{CO}_{2}$ 直接转化成 $\mathrm{CO}$ 用于 癌症治疗. 在 $630 \mathrm{~nm}$ 光照条件下, $\mathrm{AgCCN}$ 可以将 $\mathrm{CO}_{2}$ 高效地转化成 $\mathrm{CO}$, 其转换效率高达 $65 \mu \mathrm{mol} \cdot \mathrm{h}^{-1} \cdot \mathrm{g} \cdot$ $\mathrm{mat}^{-1}$. 与此同时, 在癌细胞中光催化原位生成的 CO 能

(a)

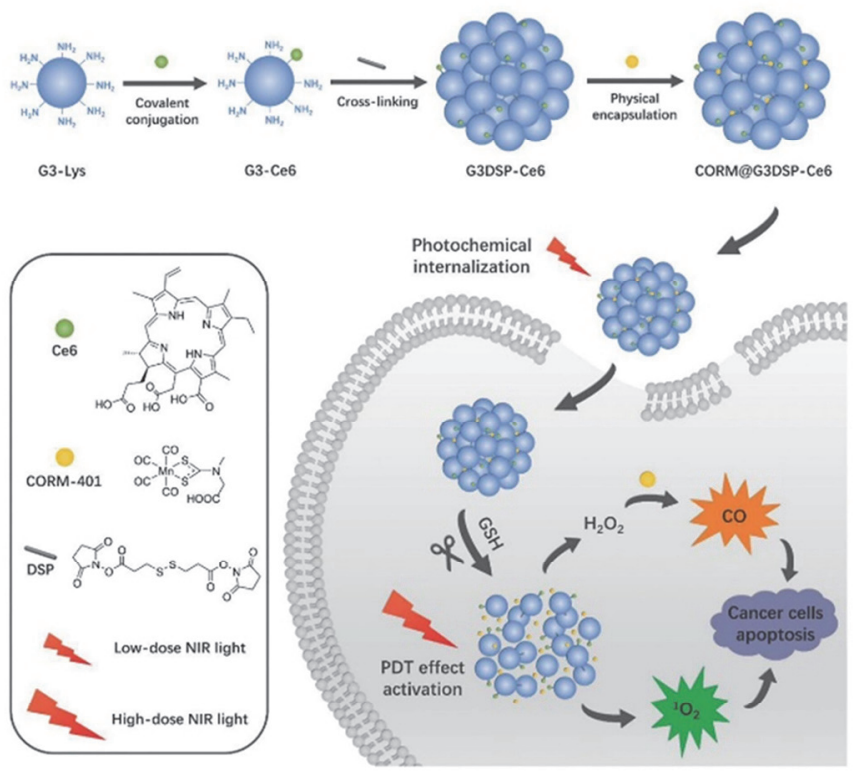

(b)
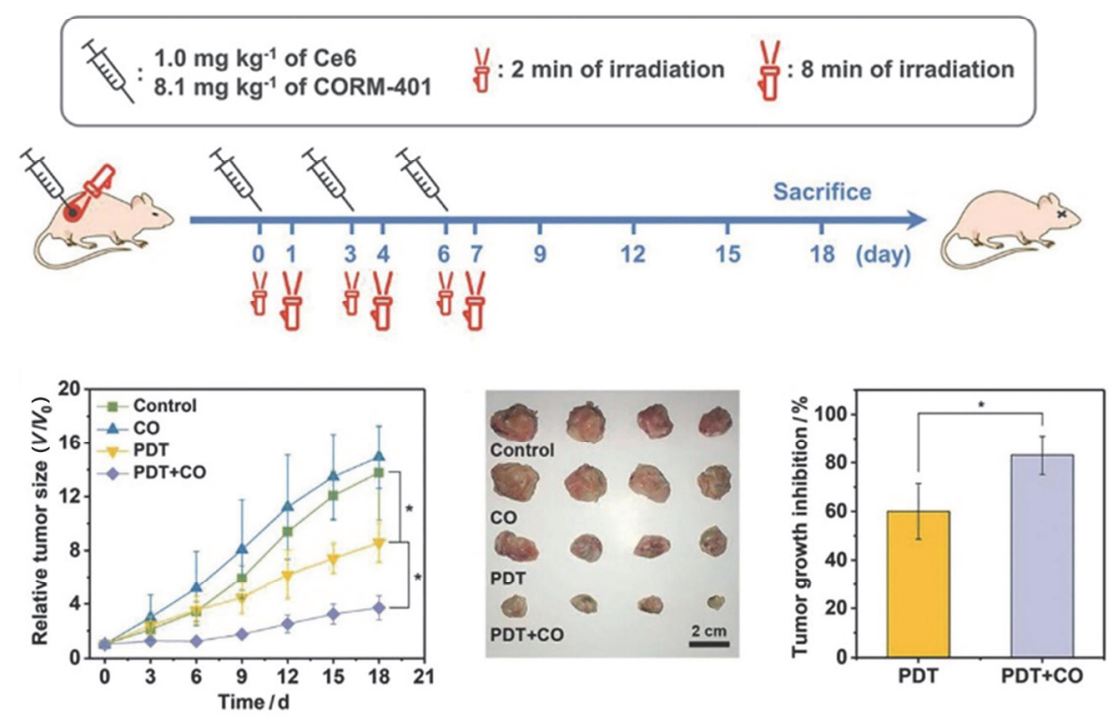

图 5 (a) CORM@G3DSP-Ce6 设计和抗肿瘤效应的示意图; (b)活体实验中肿瘤治疗的效果 ${ }^{[58]}$

Figure 5 (a) Schematic illustration for the design and anti-tumor effect of CORM@G3DSP-Ce6; (b) Efficacy of tumor therapy in vivo experiments ${ }^{[58]}$. Reproduced from Ref. 58 with permission from 2018 WILEY-VCH Verlag GmbH \& Co. KGaA, Weinheim 
(a)

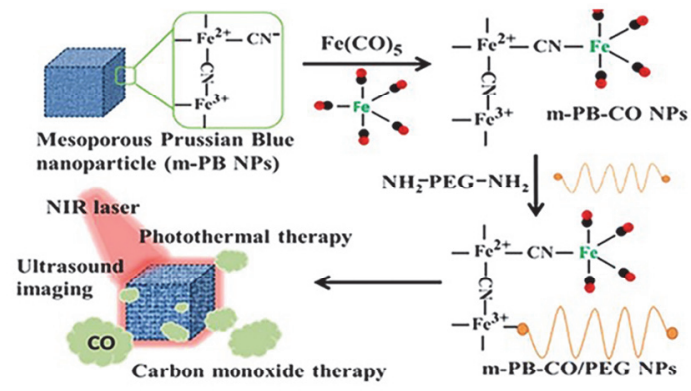

(b)

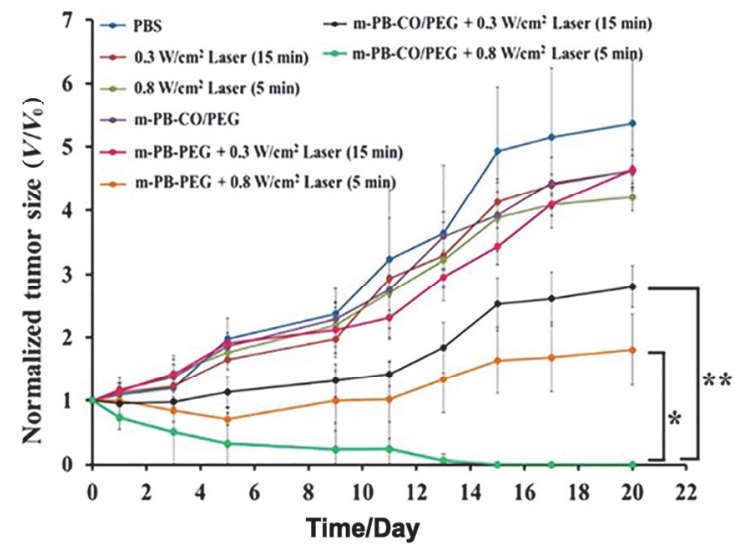

图 6 (a) 近红外光响应的 m-PB-CO/PEG 纳米颗粒的合成路径及在 光热治疗和超声成像的应用示意图; (b) 在不同处理条件下肿瘤生长 的曲线图 ${ }^{[79]}$

Figure 6 (a) Synthetic strategy for NIR-responsive m-PB-CO/PEG NPs and schematic diagram of application in photo-thermal therapy and ultrasound imaging; (b) The curve of tumor growth under different treatment conditions $^{[79]}$. Reproduced from Ref. 79 with permission from 2016 American Chemical Society

够促使肿瘤细胞线粒体的生物合成, 加剧细胞内的氧化 应激程度, 使得其对化疗药物阿霉素(DOX)更加敏感, 并保护正常细胞不受化疗药物的损伤. 体外和活体实验 都表明光催化生成的 CO 与 DOX 联合治疗具有协同抑 制肿瘤的作用. 这种能够在原位产生 $\mathrm{CO}$ 的纳米材料为 $\mathrm{CO}$ 气体在肿瘤治疗提供了新的方向.

\section{4 总结与展望}

近年来, 气体治疗作为一个新兴的研究领域在生物 医学上的应用越来越受到研究者们的关注. CO 是气体 信使分子之一, 在生物体内起到非常重要的作用. 但与 此同时, 它的生理学效应与其浓度密切相关. 如何控制 $\mathrm{CO}$ 在病灶部位的浓度成为了其在生物医学领域应用的 重要难题. 纳米材料和纳米技术的迅速发展, 为设计制 备性能优秀的可控释放型 $\mathrm{CO}$ 输运纳米材料提供了一个 良好的基础. 这篇综述详细地介绍了刺激响应型 $\mathrm{CO}$ 释 放纳米材料的最新进展, 包括由不同刺激源控制的 $\mathrm{CO}$ 释放纳米材料及其在生物医学领域的一些应用.

然而, $\mathrm{CO}$ 气体治疗仍处于一个初级阶段, 依然具有
很多问题需要进一步的研究探索. 第一, 我们需要研究 更多智能化的、能够响应不同刺激的 CO 控释纳米材料, 以满足不同疾病的需求. 尤其是针对特定分子靶点进行 响应控释的 $\mathrm{CO}$ 供体在生物医学领域具有相当重要的作 用. 我们还需要优化材料性能使得 $\mathrm{CO}$ 释放行为具有更 好可控性, 避免 $\mathrm{CO}$ 供体在血液循环系统中释放 $\mathrm{CO}$ 分 子, 以降低毒副作用. 除了通过输运供体分子释放 $\mathrm{CO}$ 外, 一些纳米材料可以在体内原位催化生成 CO. 这种 利用催化剂或纳米酶产生 $\mathrm{CO}$ 的策略与内源性 $\mathrm{CO}$ 生成 过程相似, 可以在特定部位按需释放 $\mathrm{CO}$, 具有很好的 靶向性和应用前景. 第二, $\mathrm{CO}$ 在各种疾病过程的作用和 生物学效应有待深入研究. 虽然已有很多报道称 $\mathrm{CO}$ 在 抗炎症和肿瘤治疗方面有很好的效果，但是并没有详细 地解释作用机理. 从化学的角度看, $\mathrm{CO}$ 只能与过渡金属 原子之间有相互作用, 这意味着 CO 的靶点只能是一些 特殊的生物大分子, 例如血红素等. 如果能够详细揭示 $\mathrm{CO}$ 在各种生理过程中的作用机理, 将会对 $\mathrm{CO}$ 控释材 料及其在生物医学中的应用具有重要的指导意义. 第 三, 由于 $\mathrm{CO}$ 自身有一定的毒副作用, 实时监控 $\mathrm{CO}$ 的释 放行为和浓度有非常重要的意义. 而纳米材料易于修饰 的特点决定了它可以作为一个多功能的平台, 能够同时 实现 $\mathrm{CO}$ 控释和实时浓度的监控. 借助这一平台, 研究 者们可以通过材料设计, 将 $\mathrm{CO}$ 与成像、治疗等功能结 合, 扩展 $\mathrm{CO}$ 控释纳米材料的应用领域. 第四, 纳米材 料的应用既可以为 $\mathrm{CO}$ 控释带来新的机遇也可能引入一 些风险. 研究者们需要充分考虑和研究 $\mathrm{CO}$ 控释纳米材 料的安全性问题, 包括材料摄取、积累、转化和代谢等 方面对毒性的影响. 这些都是 CO 控释纳米材料走向临 床应用的基础性问题, 有待进一步的评价和研究.

尽管还有一些尚未解决的问题, 但是 $\mathrm{CO}$ 的可控输 运及 $\mathrm{CO}$ 气体治疗仍是一个前沿的研究热点. 随着研究 的进一步深入, 特别是多学科交叉合作, $\mathrm{CO}$ 控释纳米材 料一定有着非常光明的应用前景.

\section{作者简介}

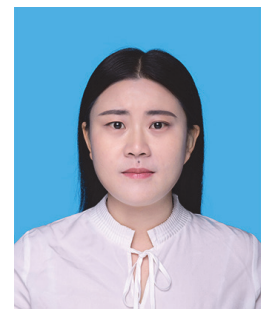

张晓蕾, 2016 年本科毕业于山东科技大学土木工程与建 筑学院; 同年进入山东科技大学, 攻读硕士学位; 2017 年 7 月, 进入中国科学院高能物理研究所纳米生物效应与安全性重点 实验室联合培养. 主要研究的方向是纳米材料的合成及生物 医学应用. 


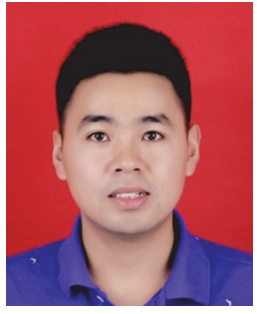

田甘, 副教授, 博士. 2015 年毕业于四川大学化学学院, 获得博士学位. 研究生期间以“协同创新培养计划”联培于中 科院纳米生物效应与安全性重点实验室, 主要研究方向为荧 光上转换纳米材料的可控合成及其生物医学研究. 2015 年, 到 陆军军医大学第一附属医院工作, 加入病理学研究所\&西南 癌症中心, 负责纳米生物医学应用平台的建设. 2017 年, 入选 陆军军医大学第五类引进人才计划, 聘为副教授.

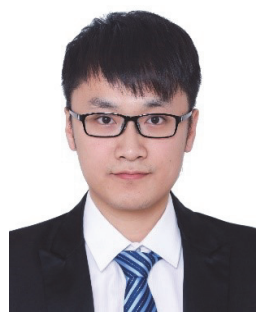

张潚, 博士后, 2018 年毕业于国家纳米科学中心, 获理学 博士学位. 2018 年 4 月起于中国科学院高能物理研究所进行博 士后研究工作. 目前从事无机纳米材料的可控合成及其生物 效应研究. 主要研究方向为气体信使小分子的可控输运及其 在肿瘤治疗中的应用.

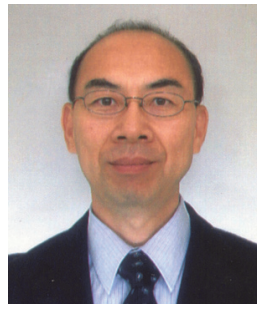

王清, 博士生导师, 1986 年本科毕业于北京大学, 1991 年 硕士毕业于山东大学, 1996 年于同济大学获得博士学位. 2013 年, 山东省泰山学者特聘教授. 现为山东科技大学纳米工程 研究所所长. 主要从事固体力学, 复合材料力学以及纳米压 印技术方面的研究.

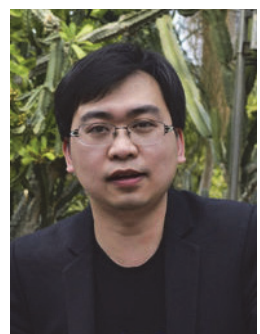

谷战军，博士生导师，国家优秀青年基金获得者. 2002 年 本科毕业于华中科技大学, 2007 年于中国科学院化学研究所 获得博士学位. 现为中国科学院高能物理研究所、中国科学院 纳米生物效应与安全性重点实验室研究员. 主要从事新型纳 米材料的可控合成及其生物效应研究.

\section{References}

[1] Queiroga, C. S.; Almeida, A. S.; Vieira, H. L. Biochem. Res. Int 2012, 2012, 749845.

[2] Haldane, J. B. S. Biochem. J. 1927, 21, 1068.

[3] Turner, M.; Hamilton-Farrell, M. R.; Clark, R. J. J. Accid. Emerg. Med. 1999, 16, 92.

[4] Untereiner, A. A.; Wu, L.; Wang, R. Gasotransmitters: Physiology and Pathophysiology, Hermann, A.; Sitdikova, G. F.; Weiger, T. M., Berlin, Heidelberg, Springer, 2012, pp. 37 70 .

[5] Coburn, R. F. N. Engl. J. Med. 1970, 282, 207.

[6] Douglas, C. G.; Haldane, J. S.; Haldane, J. B. J. Physiol. 1912, 44, 275.

[7] Slebos, D. J.; Ryter, S. W.; Choi, A. M. Respir. Res. 2003, 4, 7.

[8] Foresti, R.; Hammad, J.; Clark, J. E.; Johnson, T. R.; Mann, B. E.; Friebe, A.; Green, C. J.; Motterlini, R. Br. J. Pharmacol. 2004, 142, 453.

[9] Ma, X. L.; Sayed, N.; Beuve, A.; van den Akker, F. EMBO J. 2007, $26,578$.

[10] Rodriguez, A. I.; Gangopadhyay, A.; Kelley, E. E.; Pagano, P. J.; Zuckerbraun, B. S.; Bauer, P. M. Arterioscler. Thromb. Vasc. Biol. 2010, 30, 98 .

[11] Garcia-Gallego, S.; Bernardes, G. J. Angew. Chem., Int. Ed. 2014, $53,9712$.

[12] Donegan, S. E.; Naples, K. M. Cancer Pract. 2002, 10, 53.

[13] Ling, K.; Men, F.; Wang, W. C.; Zhou, Y. Q.; Zhang, H. W.; Ye, D. W. J. Med. Chem. 2018, 61, 2611.

[14] Motterlini, R.; Clark, J. E.; Foresti, R.; Sarathchandra, P.; Mann, B. E.; Green, C. J. Circ. Res. 2002, 90, E17.

[15] Zuckerbraun, B. S.; Chin, B. Y.; Bilban, M.; d'Avila, J. C.; Rao, J.; Billiar, T. R.; Otterbein, L. E. FASEB J. 2007, 21, 1099.

[16] Parr, S. R.; Wilson, M. T.; Greenwood, C. Biochem. J. 1975, 151 51.

[17] Brunori, M.; Parr, S. R.; Greenwood, C.; Wilson, M. T. Biochem. J. 1975, 151, 185.

[18] Gorman, D.; Drewry, A.; Huang, Y. L.; Sames, C. Toxicol. 2003, $187,25$.

[19] Pitchumony, T. S.; Spingler, B.; Motterlini, R.; Alberto, R. Chimia 2008, 62, 277.

[20] Motterlini, R.; Otterbein, L. E. Nat. Rev. Drug Discovery 2010, 9, 728.

[21] Sawle, P.; Foresti, R.; Mann, B. E.; Johnson, T. R.; Green, C. J.; Motterlini, R. Br. J. Pharmacol. 2005, 145, 800.

[22] Inaba, H.; Fujita, K.; Ueno, T. Biomater. Sci. 2015, 3, 1423.

[23] Li, Y.; Shu, Y. Z.; Liang, M. W.; Xie, X. L.; Jiao, X. Y.; Wang, X.; Tang, B. Angew. Chem. Int. Ed. 2018, 57, 12415.

[24] Sanvicens, N.; Marco, M. P. Trends Biotechnol. 2008, 26, 425.

[25] Bahrami, B.; Hojjat-Farsangi, M.; Mohammadi, H.; Anvari, E. Ghalamfarsa, G.; Yousefi, M.; Jadidi-Niaragh, F. Immunol. Lett. 2017, 190, 64.

[26] Ding, C. Z.; Li, Z. B. Mater. Sci. Eng., C 2017, 76, 1440.

[27] Wang, Z. Q.; Ciacchi, L. C.; Wei, G. Appl. Sci. 2017, 7, 1175.

[28] Gu, Z. J.; Zhu, S.; Yan, L.; Zhao, F.; Zhao, Y. L. Adv. Mater. 2018, 1800662.

[29] Kemp, J. A.; Shim, M. S.; Heo, C. Y.; Kwon, Y. J. Adv. Drug Delivery Rev. 2016, 98, 3.

[30] Blum, A. P.; Kammeyer, J. K.; Rush, A. M.; Callmann, C. E.; Hahn, M. E.; Gianneschi, N. C. J. Am. Chem. Soc. 2015, 137, 2140.

[31] Mo, R.; Gu, Z. Mater. Today 2016, 19, 274.

[32] Gulzar, A.; Gai, S. L.; Yang, P. P.; Li, C. X.; Ansari, M. B.; Lin, J. J. Mater. Chem. B 2015, 3, 8599.

[33] Swietach, P.; Vaughan-Jones, R. D.; Harris, A. L.; Hulikova, A. Phil. Trans. R. Soc. B 2014, 369, 20130099.

[34] Kato, Y.; Ozawa, S.; Miyamoto, C.; Maehata, Y.; Suzuki, A.; Maeda, T.; Baba, Y. Cancer Cell Int. 2013, 13, 89.

[35] He, Q. J. Biomater. Sci. 2017, 5, 2226.

[36] Fan, W.; Yung, B. C.; Chen, X. Angew. Chem., Int. Ed. 2018, 57, 8383.

[37] Jin, Q.; Deng, Y. Y.; Jia, F.; Tang, Z.; Ji, J. Adv. Therap. 2018 , 1800084.

[38] Yin, X. F.; Liu, X. H.; Shen, L. H.; Jin, H.; Yang, P. Y. Acta Chim. Sinica 2015，73，337. (殷薛飞，刘晓慧，申华莉，金红，杨茯原， 化学学报, 2015, 73, 337.)

[39] Kim, C. K.; Lim, S. J. Arch. Pharm. Res. 2002, 25, 229.

[40] Li, Z. T.; Yu, G. C.; Yang, J. Org. Chem. Front. 2017, 4, 115. 
[41] Zhou, L. X. Acta Chim. Sinica 2017, 75, 552. (周立祥, 化学学报, 2017, 75, 552.)

[42] Shao, W.; Liu, X.; Wang, T. T.; Hu, X. Y. Chin. J. Org. Chem. 2018, 38, 1107. (邵为, 刘昕, 王婷婷, 胡晓玉, 有机化学, 2018, 38, 1107.)

[43] Zhang, B.; Chang, B. S.; Sun, T. L. Acta Chim. Sinica 2018, 76, 35. (张蓓, 常柏松, 孙涛垒, 化学学报, 2018, 76, 35.)

[44] Li, Z. Y.; Hu, X. Y.; Jiang, J. L.; Zhang, D. M.; Xiao, S. J.; Lin, C.; Wang, L. Y. Chin. J. Org. Chem. 2018, 38, 29. (李臻益, 胡晓玉, 强 琚莉, 张冬梅, 肖守军, 林晨, 王乐勇, 有机化学, 2018, 38, 29.)

[45] Motterlini, R.; Sawle, P.; Hammad, J.; Bains, S.; Alberto, R.; Foresti, R.; Green, C. J. FASEB J. 2005, 19, 284.

[46] Chang, Y. J.; Liu, X. Z.; Zhao, Q.; Yang, X. H.; Wang, K. M.; Wang, Q.; Lin, M.; Yang, M. Chin. Chem. Lett. 2015, 26, 1203.

[47] Gu, Z.; Dang, T. T.; Ma, M.; Tang, B. C.; Cheng, H.; Jiang, S.; Dong, Y.; Zhang, Y.; Anderson, D. G. ACS Nano 2013, 7, 6758.

[48] Nguyen, D.; Adnan, N. N.; Oliver, S.; Boyer, C. Macromol. Rapid Commun. 2016, 37, 739 .

[49] Lopez-Lazaro, M. FASEB J. 2006, 20, 828.

[50] Fan, W. P.; Bu, W. B.; Shen, B.; He, Q. J.; Cui, Z. W.; Liu, Y. Y.; Zheng, X. P.; Zhao, K. L.; Shi, J. L. Adv. Mater. 2015, 27, 4155.

[51] Liu, T. P.; Wu, S. H.; Chen, Y. P.; Chou, C. M.; Chen, C. T. Nanoscale 2015, 7, 6471.

[52] Suliman, H. B.; Carraway, M. S.; Tatro, L. G.; Piantadosi, C. A. J. Cell Sci. 2007, 120, 299.

[53] Veal, E. A.; Day, A. M.; Morgan, B. A. Mol. Cell 2007, 26, 1.

[54] Tsan, M. F. Int. J. Mol. Med. 2001, 7, 13.

[55] Senturker, S.; Karahalil, B.; Inal, M.; Yilmaz, H.; Muslumanoglu, H.; Gedikoglu, G.; Dizdaroglu, M. FEBS Lett. 1997, 416, 286.

[56] Jin, Z. K.; Wen, Y. Y.; Xiong, L. W.; Yang, T.; Zhao, P. H.; Tan, L. W.; Wang, T. F.; Qian, Z. Y.; Su, B. L.; He, Q. J. Chem. Commun. 2017, 53, 5557.

[57] Jin, Z. K.; Zhao, P. H.; Zhang, J. H.; Yang, T.; Zhou, G. X.; Zhang, D. H.; Wang, T. F.; He, Q. J. Chem. Eur. J. 2018, 24, 11667.

[58] Wu, L. H.; Cai, X. J.; Zhu, H. F.; Li, J. H.; Shi, D. X.; Su, D. F.; Yue, D.; Gu, Z. W. Adv. Funct. Mater. 2018, 28, 1804324.

[59] He, Q. J.; Chen, D. Y.; Fan, M. J. J. Inorg. Mater. 2018, 33, 811. (何 前军, 陈丹阳, 范明俭, 无机材料学报, 2018, 33, 811.)

[60] Gonzales, M. A.; Han, H.; Moyes, A.; Radinos, A.; Hobbs, A. J.; Coombs, N.; Oliver, S. R. J.; Mascharak, P. K. J. Mater. Chem. B 2014, 2, 2107.

[61] Govender, P.; Pai, S.; Schatzschneider, U.; Smith, G. S. Inorg. Chem. 2013, 52, 5470.

[62] Bohlender, C.; Glaser, S.; Klein, M.; Weisser, J.; Thein, S.; Neugebauer, U.; Popp, J.; Wyrwa, R.; Schiller, A. J. Mater. Chem. B 2014, 2, 1454

[63] Bruckmann, N. E.; Wahl, M.; Reiss, G. J.; Kohns, M.; Watjen, W.; Kunz, P. C. Eur. J. Inorg. Chem. 2011, 2011, 4571.

[64] Popova, M.; Soboleva, T.; Ayad, S.; Benninghoff, A. D.; Berreau, L. M. J. Am. Chem. Soc. 2018, 140, 9721 .

[65] Fujita, K.; Tanaka, Y.; Abe, S.; Ueno, T. Angew. Chem., Int. Ed. 2016, 55, 1056.

[66] Dordelmann, G.; Meinhardt, T.; Sowik, T.; Krueger, A.; Schatzschneider, U. Chem. Commun. 2012, 48, 11528.

[67] Carmona, F. J.; Jimenez-Amezcua, I.; Rojas, S.; Romao, C. C.; Navarro, J. A. R.; Maldonado, C. R.; Barea, E. Inorg. Chem. 2017, 56,10474

[68] Chakraborty, I.; Carrington, S. J.; Hauser, J.; Oliver, S. R. J.; Mascharak, P. K. Chem. Mater. 2015, 27, 8387.

[69] Zhang, X. D.; Tian, H.; He, J. H.; Cao, Y. Acta Chim. Sinica 2013, 71, 433. (张晓丹, 田华, 贺军辉, 曹阳, 化学学报, 2013, 71, 433.)

[70] Diring, S.; Carne-Sanchez, A.; Zhang, J.; Ikemura, S.; Kim, C.; Inaba, H.; Kitagawa, S.; Furukawa, S. Chem. Sci. 2017, 8, 2381.

[71] Pierri, A. E.; Huang, P. J.; Garcia, J. V.; Stanfill, J. G.; Chui, M.; Wu, G.; Zheng, N.; Ford, P. C. Chem. Commun. 2015, 51, 2072.

[72] Askes, S. H. C.; Reddy, G. U.; Wyrwa, R.; Bonnet, S.; Schiller, A. J. Am. Chem. Soc. 2017, 139, 15292.

[73] He, Q. J.; Kiesewetter, D. O.; Qu, Y.; Fu, X.; Fan, J.; Huang, P.; Liu, Y. J.; Zhu, G. Z.; Liu, Y.; Qian, Z. Y.; Chen, X. Y. Adv. Mater. 2015, 27,6741 .

[74] Tan, M. J.; Pan, H. C.; Tan, H. R.; Chai, J. W.; Lim, Q. F.; Wong, T. I.; Zhou, X.; Hong, Z. Y.; Liao, L. D.; Kong, K. V. Adv. Healthcare Mater. 2018, 7, 1870022

[75] Wei, Z. J.; Liu, G. X.; Dong, X. T.; Wang, J. X.; Yu, W. S. Acta Chim. Sinica 2014, 72, 257. (魏忠杰, 刘桂霞, 董相廷, 王进贤,
于文生, 化学学报, 2014, 72, 257.)

[76] Zhang, X.; Guo, Z.; Liu, J.; Tian, G.; Chen, K.; Yu, S. C.; Gu, Z. J. Sci. Bull. 2017, 62, 985 .

[77] Chen, H. B.; Gu, Z. J.; An, H. W.; Chen, C. Y.; Chen, J.; Cui, R.; Chen, S. Q.; Chen, W. H.; Chen, X. S.; Chen, X. Y.; Chen, Z.; Ding, B. Q.; Dong, Q.; Fan, Q.; Fu, T.; Hou, D. Y.; Jiang, Q.; Ke, H. T.; Jiang, X. Q.; Liu, G.; Li, S. P.; Li, T. Y.; Liu, Z.; Nie, G. J.; Ovais, M.; Pang, D. W.; Qiu, N. S.; Shen, Y. Q.; Tian, H. Y.; Wang, C.; Wang, H.; Wang, Z. Q.; Xu, H. P.; Xu, J. F.; Yang, X. L.; Zhu, S.; Zheng, X. C.; Zhang, X. Z.; Zhao, Y. B.; Tan, W. H.; Zhang, X.; Zhao, Y. L. Sci. China Chem. 2018, 61, 1503.

[78] Lin, X. Y.; Wang, J. Acta Chim. Sinica 2017, 75, 979 (in Chinese). (林㴋羽, 王璟, 化学学报, 2017, 75, 979.)

[79] Li, W. P.; Su, C. H.; Tsao, L. C.; Chang, C. T.; Hsu, Y. P.; Yeh, C. S. ACS Nano 2016, 10, 11027.

[80] Cole, A. J.; Yang, V. C.; David, A. E. Trends Biotechnol. 2011, 29, 323.

[81] Williams, P. S.; Carpino, F.; Zborowski, M. Mol. Pharmaceutics 2009, 6, 1290.

[82] Pankhurst, Q. A.; Connolly, J.; Jones, S. K.; Dobson, J. J. Phys. D: Appl. Phys. 2003, 36, R167.

[83] Kunz, P. C.; Meyer, H.; Barthel, J.; Sollazzo, S.; Schmidt, A. M.; Janiak, C. Chem. Commun. 2013, 49, 4896.

[84] Meyer, H.; Winkler, F.; Kunz, P.; Schmidt, A. M.; Hamacher, A.; Kassack, M. U.; Janiak, C. Inorg. Chem. 2015, 54, 11236.

[85] Stone, J. R.; Marletta, M. A. Biochemistry 1994, 33, 5636.

[86] Botros, F. T.; Navar, L. G. Am. J. Physiol. Heart Circ. Physiol. 2006, 291, H2772.

[87] Ramos, K. S.; Lin, H.; McGrath, J. J. Biochem. Pharmacol. 1989, $38,1368$.

[88] Li, A. L.; Xi, Q.; Umstot, E. S.; Bellner, L.; Schwartzman, M. L.; Jaggar, J. H.; Leffler, C. W. Circ. Res. 2008, 102, 234.

[89] Song, Y. C.; Liu, J. X.; Zhang, Y. Y.; Shi, W.; Ma, H. M. Acta Chim. Sinica 2013，71，1607. (宋延超，刘俊秀，张阳阳，史文，马会民, 化学学报, 2013, 71, 1607.)

[90] Otterbein, L. E.; Bach, F. H.; Alam, J.; Soares, M.; Lu, H. T.; Wysk, M.; Davis, R. J.; Flavell, R. A.; Choi, A. M. Nat. Med. 2000, 6, 422.

[91] Lee, T. S.; Tsai, H. L.; Chau, L. Y. J. Biol. Chem. 2003, 278, 19325.

[92] Nguyen, D.; Nguyen, T. K.; Rice, S. A.; Boyer, C. Biomacromolecules 2015, 16, 2776.

[93] Motterlini, R.; Mann, B. E.; Foresti, R. Expert Opin. Investig. Drugs 2005, 14, 1305 .

[94] Mann, B. E. Medicinal Organometallic Chemistry. Topics in Organometallic Chemistry, Eds.: Jaouen, G.; Metzler-Nolte, N., Berlin, Heidelberg, Springer, 2010, Vol. 32, p. 247.

[95] Ferrandiz, M. L.; Maicas, N.; Garcia-Arnandis, I.; Terencio, M. C.; Motterlini, R.; Devesa, I.; Joosten, L. A.; van den Berg, W. B.; Alcaraz, M. J. Ann. Rheum. Dis. 2008, 67, 1211.

[96] Bathoorn, E.; Slebos, D. J.; Postma, D. S.; Koeter, G. H.; van Oosterhout, A. J.; van der Toorn, M.; Boezen, H. M.; Kerstjens, H. A. Eur. Respir. J. 2007, 30, 1131.

[97] Nowick, J. S.; Chung, D. M.; Maitra, K.; Maitra, S.; Stigers, K. D.; Sun, Y. J. Am. Chem. Soc. 2000, 122, 7654.

[98] Morse, D.; Pischke, S. E.; Zhou, Z.; Davis, R. J.; Flavell, R. A.; Loop, T.; Otterbein, S. L.; Otterbein, L. E.; Choi, A. M. J. Biol. Chem. 2003, 278, 36993.

[99] Otterbein, L. E.; Choi, A. M. Am. J. Physiol. Lung Cell Mol. Physiol. 2000, 279, L1029.

[100] Pae, H. O.; Oh, G. S.; Choi, B. M.; Chae, S. C.; Kim, Y. M.; Chung, K. R.; Chung, H. T. J. Immunol. 2004, 172, 4744.

[101] Song, R. P.; Zhou, Z. H.; Kim, P. K.; Shapiro, R. A.; Liu, F.; Ferran, C.; Choi, A. M.; Otterbein, L. E. J. Biol. Chem. 2004, $279,44327$.

[102] Bani-Hani, M. G.; Greenstein, D.; Mann, B. E.; Green, C. J.; Motterlini, R. J. Pharmacol. Exp. Ther. 2006, 318, 1315.

[103] Bani-Hani, K. E.; Bani-Hani, B. K. World J. Gastroenterol. 2006, 12,1521 .

[104] Guillen, M. I.; Megias, J.; Clerigues, V.; Gomar, F.; Alcaraz, M. J. Rheumatol. 2008, 47, 1323.

[105] Hasegawa, U.; van der Vlies, A. J.; Simeoni, E.; Wandrey, C.; Hubbell, J. A. J. Am. Chem. Soc. 2010, 132, 18273.

[106] Van der Vlies, A. J.; Inubushi, R.; Uyama, H.; Hasegawa, U. Bioconjug. Chem. 2016, 27, 1500

[107] Qureshi, O. S.; Zeb, A.; Akram, M.; Kim, M. S.; Kang, J. H.; Kim, H. S.; Majid, A.; Han, I.; Chang, S. Y.; Bae, O. N.; Kim, J. K. Eur. J. Pharm. Biopharm. 2016, 108, 187. 
[108] Fujita, K.; Tanaka, Y.; Sho, T.; Ozeki, S.; Abe, S.; Hikage, T.; Kuchimaru, T.; Kizaka-Kondoh, S.; Ueno, T. J. Am. Chem. Soc. 2014, 136, 16902.

[109] Fujita, K.; Tanaka, Y.; Abe, F.; Ueno, T. Angew. Chem., Int. Ed. 2016, 55, 1056.

[110] Nobre, L. S.; Seixas, J. D.; Romao, C. C.; Saraiva, L. M. Antimicrob. Agents Chemother. 2007, 51, 4303.

[111] Lu, Y.; Slomberg, D. L.; Schoenfisch, M. H. Biomaterials 2014, 35, 1716.

[112] Lu, Y.; Slomberg, D. L.; Shah, A.; Schoenfisch, M. H. Biomacromolecules 2013, 14, 3589.

[113] Murray, T. S.; Okegbe, C.; Gao, Y.; Kazmierczak, B. I.; Motterlini, R.; Dietrich, L. E.; Bruscia, E. M. PLoS One 2012, 7, e35499.

[114] Nobre, L. S.; Al-Shahrour, F.; Dopazo, J.; Saraiva, L. M. Microbiology 2009, 155, 813 .

[115] Desmard, M.; Davidge, K. S.; Bouvet, O.; Morin, D.; Roux, D.; Foresti, R.; Ricard, J. D.; Denamur, E.; Poole, R. K.; Montravers, P.; Motterlini, R.; Boczkowski, J. FASEB J. 2009, 23, 1023.

[116] Loboda, A.; Jazwa, A.; Wegiel, B.; Jozkowicz, A.; Dulak, J. Cell Mol. Biol. (Noisy-le-grand) 2005, 51, 347.

[117] Chung, S. W.; Liu, X. L.; Macias, A. A.; Baron, R. M.; Perrella, M. A. J. Clin. Invest. 2008, 118, 239.
[118] Bang, C. S.; Kruse, R.; Johansson, K.; Persson, K. BMC Microbiology 2016, 16, 64 .

[119] Flanagan, L.; Steen, R. R.; Saxby, K.; Klatter, M.; Aucott, B. J.; Winstanley, C.; Fairlamb, I. J. S.; Lynam, J. M.; Parkin, A.; Friman, V.-P. Front. Microbiol. 2018, 9, 195.

[120] Wilson, J. L.; Jesse, H. E.; Poole, R. K.; Davidge, K. S. Curr. Pharm. Biotechnol. 2012, 13, 760 .

[121] Li, B.; Zhang, X. Y.; Yang, J. Z.; Zhang, Y. J.; Li, W. X.; Fan, C. H.; Huang, Q. Int. J. Nanomed. 2014, 9, 4697.

[122] Folkman, J. N. Engl. J. Med. 1971, 285, 1182.

[123] Calderon-Montano, J. M.; Burgos-Moron, E.; Orta, M. L.; Mateos, S.; Lopez-Lazaro, M. Planta Med. 2013, 79, 1017.

[124] Pompella, A.; Visvikis, A.; Paolicchi, A.; De Tata, V.; Casini, A. F. Biochem. Pharmacol. 2003, 66, 1499.

[125] Wu, X. Y.; Zhang, L.; Lü, D.; Liu, Y. H.; Chen, Y. N.; Su, W. J.; Luo, N.; Xiang, R. Acta Chim. Sinica 2013, 71, 299. (吴星怡, 张磊, 吕 丹, 刘艳华，陈亚南，苏位君，罗娜，向荣，化学学报，2013，71, 299.)

[126] Matsumura, Y.; Maeda, H. Cancer Res. 1986, 46, 6387.

[127] Zheng, D. W.; Li, B.; Li, C. X.; Xu, L.; Fan, J. X.; Lei, Q.; Zhang, X. Z. Adv. Mater. 2017, 29, 1703822. 